\title{
A Game Theoretic Analysis of Incentives in Content Production and Sharing over Peer-to-Peer Networks
}

\author{
Jaeok Park and Mihaela van der Schaar
}

\begin{abstract}
User-generated content can be distributed at a low cost using peer-to-peer (P2P) networks, but the free-rider problem hinders the utilization of P2P networks. In order to achieve an efficient use of P2P networks, we investigate fundamental issues on incentives in content production and sharing using game theory. We build a basic model to analyze non-cooperative outcomes without an incentive scheme and then use different game formulations derived from the basic model to examine five incentive schemes: cooperative, payment, repeated interaction, intervention, and enforced full sharing. The results of this paper show that 1) cooperative peers share all produced content while non-cooperative peers do not share at all without an incentive scheme; 2) a cooperative scheme allows peers to consume more content than non-cooperative outcomes do; 3) a cooperative outcome can be achieved among non-cooperative peers by introducing an incentive scheme based on payment, repeated interaction, or intervention; and 4) enforced full sharing has ambiguous welfare effects on peers. In addition to describing the solutions of different formulations, we discuss enforcement and informational requirements to implement each solution, aiming to offer a guideline for protocol designers when designing incentive schemes for P2P networks.
\end{abstract}

\section{Index Terms}

Game theory, incentives, network economics, peer-to-peer networks, pricing schemes.

The authors are with Electrical Engineering Department, University of California, Los Angeles (UCLA), 420 Westwood Plaza, Los Angeles, CA 90095-1594, USA. e-mail: \{jaeok, mihaela $\} @$ ee.ucla.edu. 


\section{INTRODUCTION}

Recent developments in technology have significantly reduced the cost of producing and distributing content in various forms such as images, sounds, videos, and text. Once produced only by companies with a large capital, content can now be produced by end-users. In today's Internet-based social communities, peer-to-peer (P2P) networks offer a cost effective and easily deployable framework for sharing usergenerated content [1]. While P2P networks have many advantages such as scalability, resilience, and effectiveness in coping with dynamics and heterogeneity [2], they are vulnerable to intrinsic incentive problems in that the transfer of content incurs costs to uploaders as well as to downloaders but benefits only downloaders. Since the social cost of transfer (the sum of upload and download costs) exceeds the private cost of transfer (download costs), peers tend to download excessibly as in the tragedy of the commons problem. On the other hand, since upload incurs costs to uploaders without giving them direct benefit, peers tend to upload too little. The incentive problem stating that peers desire to benefit from P2P networks while not contributing to them is referred to as the free-rider (or freeloader) problem.

Various incentive schemes to mitigate the free-rider problem have been proposed and analyzed in the literature. Cooperative schemes (e.g., [3], [4]) utilize helpers that download files on behalf of a peer in the same collaborative group. Helpers can improve the download performance of P2P networks by sharing their spare upload capacities. However, forming and sustaining collaborative groups in a distributed system poses a main challenge to cooperative schemes. Payment schemes (e.g., [5], [6]) use virtual currency or micropayment to reward upload and charge download. Payment schemes have a solid theoretical foundation as they are based on economic models. However, they are regarded impractical because they require an accounting infrastructure to track the transactions of peers [7]. Differential service schemes (e.g., [8], [9]) allow peers to make upload decisions based on the rating of a peer that requests content from them. Since a peer with a good reputation is treated preferentially by other peers, differential service schemes provide incentives for peers to contribute in order to build and maintain a good reputation. However, differential service schemes require large communication overheads to determine and announce the ratings of peers. The rating of a peer is determined by its past actions, which are observed by different peers, and peers need to know the rating of every other peer that they interact with.

Game theory [10] offers a useful framework to model multi-user interaction and has been applied to analyze the behavior of peers in P2P networks. Incentive schemes such as payment schemes and differential service schemes have been investigated using non-cooperative game theory. Payment schemes can be easily incorporated in static game models as in [11], while differential service schemes have been 
studied in the context of different game models. [7] uses a static game model to analyze the steadystate outcome of learning dynamics under a differential service scheme. [12] simulates an evolutionary game model to examine the performance of a differential service scheme based on peer reciprocation. [13] uses a repeated game model to construct a differential service scheme based on the idea of social norms [14]. [15] and [16] apply the mechanism design approach to build optimal incentive-compatible differential service schemes. [17] uses both repeated game and mechanism design approaches to propose cheat-proof and attack-resistant differential service schemes. Cooperative game theory has also been used to investigate coalition formation among peers [18], [19].

In this paper, we investigate fundamental issues on incentives in content production and sharing over P2P networks using game theory. Unlike existing game-theoretic works on P2P networks, which focus on a particular game model to construct incentive schemes, we build a basic model and use it as a unified framework based on which different incentive schemes are examined applying various game theoretic models. Specifically, we analyze the basic model as a non-cooperative game and examine five incentive schemes - cooperative, payment, repeated interaction, intervention, and enforced full sharing — using different game formulations derived from the basic model, as summarized in Table I. Hence, instead of arguing for a particular incentive scheme and a modeling approach, we show that alternative incentive schemes can provide incentives for sharing in P2P networks from a neutral perspective. As can be seen from Table I, different incentive schemes and the corresponding game models have different requirements for implementation. Since the characteristics of P2P networks vary depending on their architecture, the effectiveness of an incentive scheme will depend on the network environment. Thus, our analysis in this paper can serve as a guideline for a protocol designer when modeling, comparing, and selecting incentive schemes.

Another distinctive feature of our framework is that we allow peers to make production decisions whereas most of existing works assume that peers are endowed with a certain amount of content (see, for example, [11], [16]). When produced content and downloaded content are substitutable in consumption, the amount of content a peer produces is affected by the amount of content available in a P2P network. By endogenizing the amount of content that peers produce, we can capture the strategic link between producing and downloading content. In addition, we consider scenarios where the sharing decisions of peers can be enforced while production and download decisions are made in a non-cooperative manner. These scenarios can be formulated as games with partial cooperation in which the strategies of players can be enforced only in some stages. The concepts developed in the discussion of enforced sharing decisions can be applied to other scenarios that can be modeled as multi-stage games. 
TABLE I: Comparisons of the approaches discussed in the paper. $\mathbf{v} \triangleq\left(v_{1}, \ldots, v_{N}\right)$ represents the utility functions of peers. $\mathbf{y}^{*} \triangleq\left(y_{1}^{*}, \ldots, y_{N}^{*}\right)$ represents the Pareto efficient sharing levels of peers desired by the protocol designer (PD). $t^{*}$ and $q^{*}$ represent optimal payment and intervention schemes, respectively, that implements $\mathbf{y}^{*}$. Rationality of peers are assumed throughout.

\begin{tabular}{|c|c|c|c|c|}
\hline Section & Approach & Requirements (enforcement and information) & Pareto efficiency & Prop. \\
\hline III & Non-cooperative & There are no requirements for the PD and peers. & Inefficient & 1 \\
\hline IV & Cooperative & $\begin{array}{l}\text { The PD knows } \mathbf{v} \text { to compute } \mathbf{y}^{*} \text {. } \\
\text { The PD enforces } \mathbf{y}^{*} \text {. } \\
\text { Peer } i \text { knows } \mathbf{y}^{*} \text {. }\end{array}$ & Efficient & 4 \\
\hline V & Payment & $\begin{array}{l}\text { The PD knows } \mathbf{v} \text { to compute } t^{*} \text {. } \\
\text { The PD enforces } t^{*} \text {. } \\
\text { Peer } i \text { knows } t^{*} \text {. }\end{array}$ & Efficient & 6 \\
\hline VI.A & $\begin{array}{l}\text { Differential service } \\
\text { based on } \\
\text { repeated interaction }\end{array}$ & $\begin{array}{l}\text { The PD knows } \mathbf{v} \text { to compute } \mathbf{y}^{*} \text {. } \\
\text { Peer } i \text { knows } \mathbf{y}^{*} \text { and the punishment rule, } \\
\text { and maintains a history of past observations. }\end{array}$ & Efficient & 7 \\
\hline VI.B & $\begin{array}{l}\text { Differential service } \\
\text { based on } \\
\text { intervention }\end{array}$ & $\begin{array}{l}\text { The PD knows } \mathbf{v} \text { to compute } q^{*} \text {. } \\
\text { The PD enforces } q^{*} \text {. } \\
\text { Peer } i \text { knows } q^{*} \text {. }\end{array}$ & Efficient & 8 \\
\hline VII & $\begin{array}{l}\text { Enforced } \\
\text { full sharing }\end{array}$ & $\begin{array}{l}\text { The PD enforces full sharing (not sharing levels). } \\
\text { Peer } i \text { knows } \mathbf{v} \text { for endogenous network formation. }\end{array}$ & Inefficient & 9,11 \\
\hline
\end{tabular}

The rest of this paper is organized as follows. In Section II, we formulate the basic model that describes a scenario of content production and sharing. In Section III, we analyze the basic model as a noncooperative game and identify the free-rider problem. In Section IV, we investigate cooperative schemes by deriving a coalitional game based on the basic model. In Section V, we augment the basic model with a payment scheme to achieve cooperative outcomes among non-cooperative peers. In Section VI, we study differential service schemes applying repeated game and intervention approaches to the basic model. In Section VII, we analyze a partially cooperative scenario where full sharing is enforced. In Section VIII, we provide numerical illustration. In Section IX, we conclude and discuss future directions. Proofs of propositions are provided either following propositions or in Appendix B.

\section{MODEL}

We consider a completely connected P2P network of $N$ peers as in [7], [11]. Peers produce content (e.g., photos, videos, news, and customer reviews) and use the P2P network to distribute produced content. Following [20], we model the content production and sharing scenario as a sequential game consisting of three stages, which is called the content production and sharing (CPS) game. 
- Stage One (Production): Each peer determines its level of production $1 x_{i} \in \mathbb{R}_{+}$represents the amount of content produced by peer $i$ and is known only to peer $i$.

- Stage Two (Sharing): Each peer specifies its level of sharing. $y_{i} \in\left[0, x_{i}\right]$ represents the amount of content that peer $i$ makes available to other peers. $\left(y_{1}, \ldots, y_{N}\right)$ is known to all peers at the end of stage two.

- Stage Three (Transfer): Each peer determines the amounts of content that it downloads from other peers. Peer $i$ serves all the requests it receives from any other peer up to $y_{i} . z_{i j} \in\left[0, y_{j}\right]$ represents the amount of content that peer $i$ downloads from peer $j \neq i$, or equivalently peer $j$ uploads to peer $i$.

Let $\mathcal{N} \triangleq\{1, \ldots, N\}$ be the set of peers in the P2P network. For notations, we define $\mathbf{x} \triangleq\left(x_{1}, \ldots, x_{N}\right)$, $\mathbf{y} \triangleq\left(y_{1}, \ldots, y_{N}\right)$, and $\mathbf{Z} \triangleq\left[z_{i j}\right]_{i, j \in \mathcal{N}}$, an $N$-by- $N$ matrix whose $(i, j)$-entry is given by $z_{i j}$, where we set $z_{i i}=0$ for all $i \in \mathcal{N}$. The download profile of peer $i$ is given by the $i$ th row of $\mathbf{Z}$, denoted by $\mathbf{z}_{i} \triangleq\left(z_{i 1}, \ldots, z_{i N}\right)$. Similarly, the upload profile of peer $i$ is given by the $i$ th column of $\mathbf{Z}$, denoted by $\mathbf{z}^{i} \triangleq\left(z_{1 i}, \ldots, z_{N i}\right)$. Given the elements of $\mathbf{Z}$, we can compute the download volume of peer $i$ by $d_{i}\left(\mathbf{z}_{i}\right) \triangleq \sum_{j=1}^{N} z_{i j}$ and its upload volume by $u_{i}\left(\mathbf{z}^{i}\right) \triangleq \sum_{j=1}^{N} z_{j i}$. For notational convenience, we suppress the dependence of $d_{i}$ and $u_{i}$ on $\mathbf{Z}$ and write $d_{i}$ and $u_{i}$ instead of $d_{i}\left(\mathbf{z}_{i}\right)$ and $u_{i}\left(\mathbf{z}^{i}\right)$, respectively. Also, we define $w(\mathbf{Z})$ to be the total transfer volume of the P2P network given $\mathbf{Z}$, i.e., $w(\mathbf{Z}) \triangleq \sum_{i=1}^{N} \sum_{j=1}^{N} z_{i j}=$ $\sum_{i=1}^{N} d_{i}=\sum_{i=1}^{N} u_{i}$, which can be considered as a measure of the utilization of the P2P network.

We assume that peers produce nonidentical content of homogeneous quality, which allows us to focus on the quantity of content. The total amount of content that peer $i$ has at the end of the CPS game, which we call the consumption of peer $i$, is given by the sum of the amounts it produces and downloads, $x_{i}+d_{i}$. The utility of peer $i$ is given by the benefit of consumption minus the costs of production, download, and upload:

$$
v_{i}(\mathbf{x}, \mathbf{y}, \mathbf{Z})=f\left(x_{i}+d_{i}\right)-\kappa x_{i}-\delta d_{i}-\sigma u_{i} .
$$

We analyze the case of homogeneous peers in that $f, \kappa, \delta$, and $\sigma$ are the same for all peers. 2 The benefit of consumption is measured by a concave function $f: \mathbb{R}_{+} \rightarrow \mathbb{R}_{+}$as in [21]. We assume that $f$ is twice continuously differentiable and satisfies $f(0)=0, f^{\prime}>0$ and $f^{\prime \prime}<0$ on $\mathbb{R}_{++}$. We also assume

\footnotetext{
${ }^{1}$ We use the term production in a broad sense to mean any method of obtaining content other than download in the P2P network.

${ }^{2}$ We consider homogeneous peers for analytic tractability. The concepts in this paper can be extended to the case with heterogeneous peers in a straightforward manner.
} 
that $f^{\prime}(0)$ is finite $3 f^{\prime}(0)>\kappa$, and $\lim _{x \rightarrow \infty} f^{\prime}(x)=0$ so that for every $\alpha \in\left(0, f^{\prime}(0)\right]$ there exists a unique $\hat{x}_{\alpha} \geq 0$ that satisfies $f^{\prime}\left(\hat{x}_{\alpha}\right)=\alpha$. We use linear cost functions as widely adopted in the literature (see, for example, [7], [22]) 4 The cost of producing the amount of content $x_{i}$ is given by $\kappa x_{i}$, where $\kappa>0$ is the marginal cost of production. Download and upload create costs in terms of bandwidth usage, and transferring the amount of content $z_{i j}$ from peer $i$ to peer $j$ induces a cost of $\delta z_{i j}$ to peer $i$ (the downloader) and $\sigma z_{i j}$ to peer $j$ (the uploader), where $\delta>0$ and $\sigma>0$ are the marginal costs of download and upload, respectively. The P2P network has a positive social value only if obtaining a unit of content through the P2P network costs less to peers than producing it privately. Hence, we assume that $\kappa>\delta+\sigma$ to ensure that the $\mathrm{P} 2 \mathrm{P}$ network is socially valuable.

We illustrate the considered scenario with an example of financial data. Suppose that peers need financial data (e.g., earnings of companies, gross domestic products, and interest rates) in order to make forecasts based on which they make investment decisions (e.g., trade stocks and bonds). To obtain financial data, peers can either collect data by themselves or download data shared by other peers. A peer can make a more informed decision with a larger amount of data. Hence, benefits that peers receive from data can be represented by an increasing benefit function. The benefit function is concave when the marginal returns of information are diminishing in the total amount. Alternatively, we can obtain a concave benefit function by assuming that there is possible duplication in collected data, peers cannot identify the contents of data before downloading them, and the benefit is proportional to the amount of distinct data. Appendix A presents a formal proof of this statement.

\section{NON-COOPERATIVE ANALYSIS}

We first study the non-cooperative outcome of the CPS game, without any incentive or enforcement device. Non-cooperative peers choose their strategies to maximize their own utilities given others' strategies. Thus, peers' strategies should be self-enforcing at non-cooperative equilibrium in that no peer can gain by choosing a different strategy unilaterally. A strategy for peer $i$ in the CPS game is its complete contingent plan over the three stages and is denoted by $\left(x_{i}, y_{i}\left(x_{i}\right), \mathbf{z}_{i}\left(x_{i}, \mathbf{y}\right)\right)$. A stage-one strategy for peer $i$ is represented by $x_{i} \in \mathbb{R}_{+}$, a stage-two strategy by a function $y_{i}: \mathbb{R}_{+} \rightarrow \mathbb{R}_{+}$such that $y_{i}\left(x_{i}\right) \leq x_{i}$ for all $x_{i} \in \mathbb{R}_{+}$, and a stage-three strategy by a function $\mathbf{z}_{i}: \mathcal{I}_{3} \rightarrow \mathbb{R}_{+}^{N}$ such that $z_{i j}\left(x_{i}, \mathbf{y}\right) \leq y_{j}$ for all

\footnotetext{
${ }^{3}$ We use $f^{\prime}(0)$ to represent the right derivative of $f$ at 0 .

${ }^{4}$ The linearity of cost functions is assumed for analytic convenience as it allows us to obtain closed-form expressions for production levels at various solution concepts. The results of this paper can be easily extended to the case of a general convex cost function of production, as discussed in Sections III, IV, and V.
} 
$j \neq i$ and $z_{i j}\left(x_{i}, \mathbf{y}\right)=0$ for $j=i$, where $\mathcal{I}_{3} \triangleq\left\{\left(x_{i}, \mathbf{y}\right) \mid x_{i} \in \mathbb{R}_{+}, y_{i} \in\left[0, x_{i}\right], y_{j} \in \mathbb{R}_{+}, \forall j \neq i\right\}$ is the set of possible information sets at the beginning of stage three.

Nash equilibrium (NE) of the CPS game is defined as a strategy profile such that no peer can improve its utility by a unilateral deviation. The play on the equilibrium path at an NE is called an NE outcome of the CPS game. A refinement of NE for sequential games is subgame-perfect equilibrium (SPE), which requires that players choose NE strategies in any subgame, thereby eliminating incredible threats. Subgame perfection provides robustness in equilibrium strategies in that deviation is unprofitable not only at the beginning of the game but also at any stage of the game. However, formally there is no subgame of the CPS game starting from stage two or three because the stage-one choice of a peer is not revealed to other peers. Hence, SPE fails to provide a refinement of NE in the CPS game.

In order to extend the spirit of subgame perfection to non-singleton information sets, we can use sequential rationality, which postulates that players behave optimally in each information set for a given belief system [10]. Sequential rationality is required by the solution concepts of perfect Bayesian equilibrium (PBE) and sequential equilibrium (SE) 5 The difference between these two solution concepts disappears in the CPS game because the consistent belief of peer $i$ on $\mathbf{x}_{-i} \triangleq\left(x_{1}, \ldots, x_{i-1}, x_{i+1}, \ldots, x_{N}\right)$ should be the correct $\mathbf{x}_{-i}$ in both solution concepts. Hence, we use SE to refer to a solution concept requiring sequential rationality and specify only the strategy profile to describe SE suppressing the belief system with an implicit premise that peers hold correct beliefs 6 An SE strategy profile of the CPS game can be found applying a backward induction argument, which is described in detail in [20]. As with NE, the play on the equilibrium path at an SE is called an SE outcome of the CPS game.

Proposition 1. At the unique SE outcome of the CPS game, we have $x_{i}=\hat{x}_{\kappa}, y_{i}=0, \mathbf{z}_{i}=(0, \ldots, 0)$ for all $i \in \mathcal{N}$. Thus, $w(\mathbf{Z})=0$ at $S E$.

Proof: A formal proof can be found in [20, Prop. 1]. Since sharing can incur the cost of upload while it gives no benefit to the sharing peer, it is never optimal for a peer to share a positive amount. Expecting no sharing, each peer produces the autarkic optimal amount of content, $\hat{x}_{\kappa}$, which maximizes

\footnotetext{
${ }^{5}$ A strategy profile and a belief system constitute a PBE if the strategies are sequentially rational given the belief system and the beliefs are updated using Bayes' rule, whenever possible, given the strategy profile. SE is a refinement of PBE in that SE perturbs the strategy profile to make Bayes' rule applicable in every information set. See [10] for the formal definitions of PBE and SE.

${ }^{6}$ This requirement can be relaxed using the notion of self-confirming equilibrium (SCE) [23], which requires only observational consistency in beliefs.
} 
$f(x)-\kappa x$.

Remark. Suppose that the cost of production is given by a general function $c\left(x_{i}\right)$ instead of $\kappa x_{i}$. There is still no sharing at SE, but each peer produces an amount that maximizes $f(x)-c(x)$, assuming that a maximum exists.

Proposition 1 shows that when peers behave non-cooperatively, even a small cost of upload makes the socially valuable P2P network never utilized because peers are not compensated for their upload. This result explains the free-riding behavior of peers in file sharing P2P networks such as Napster and Gnutella, as reported in [24], [25]. Using a similar argument as in the formal proof of Proposition 1, we can show that the NE outcome of the CPS game is the same as the SE outcome. NE may prescribe suboptimal strategies off the equilibrium path, but there cannot be a positive amount shared on the equilibrium path 7 Individual utility and total utility at non-cooperative equilibrium are $f^{*}(\kappa)$ and $\Pi^{N C}=N f^{*}(\kappa)$, respectively, where we define $f^{*}(\alpha)=\sup _{x \geq 0}\{f(x)-\alpha x\}$ for $\alpha \in \mathbb{R}$ as the conjugate of $f$ [26] 8

\section{CoOperative Schemes}

We consider cooperative schemes in the CPS game, which allow peers to form collaborative groups and to maximize their joint welfare. In order to prevent peers from behaving non-cooperatively, cooperative schemes need to enforce the actions of peers by a contract or a protocol. The protocol designer can implement a cooperative scheme if he knows the utility functions of all peers in order to determine a desired operating point and can enforce the operating point. An example of P2P networks to which a cooperative scheme can be applied is a camera network, where cameras in different locations capture the images of an object from various angles. A property that a desired operating point should possess is Pareto efficiency (PE), which is satisfied when there is no other operating point that makes some peers better off without making other peers worse off. We define social welfare by the sum of the utilities of peers, i.e., $\Pi(\mathbf{x}, \mathbf{y}, \mathbf{Z}) \triangleq \sum_{i=1}^{N} v_{i}(\mathbf{x}, \mathbf{y}, \mathbf{Z})$. Then an allocation is Pareto efficient (PE) if it maximizes social welfare among feasible allocations 9

Proposition 2. Let $\beta \triangleq \frac{1}{N} \kappa+\frac{N-1}{N}(\delta+\sigma)$. At PE, we have $\sum_{i=1}^{N} x_{i}=\hat{x}_{\beta}$ and $x_{i}=y_{i}=z_{j i}$ for all $j \neq i$, for all $i \in \mathcal{N}$. Thus, $w(\mathbf{Z})=(N-1) \hat{x}_{\beta}$ at $P E$.

\footnotetext{
${ }^{7}$ A similar remark holds for SCE. As soon as a peer shares its content, it learns that others request its content, and thus it will choose not to share at all in order to avoid upload costs.

${ }^{8}$ Note that the definition of a conjugate is adjusted as $f$ is a concave function.

${ }^{9}$ An allocation $(\mathbf{x}, \mathbf{y}, \mathbf{Z})$ is feasible if $x_{i} \geq 0,0 \leq y_{i} \leq x_{i}, z_{i i}=0$, and $0 \leq z_{i j} \leq y_{j}$ for all $j \neq i$, for all $i \in \mathcal{N}$.
} 
Proof: A formal proof can be found in [20, Prop. 2]. PE can occur only when peers share all produced content and download all shared content. Then the social welfare maximization problem can be written as

$$
\max _{\mathbf{x} \geq 0} N f\left(\sum_{i=1}^{N} x_{i}\right)-[\kappa+(N-1)(\delta+\sigma)] \sum_{i=1}^{N} x_{i} .
$$

The first-order optimality condition for $X \triangleq \sum_{i=1}^{N} x_{i}$ is $f^{\prime}(X)=\beta$. Note that $\beta$ is the per capita marginal cost of obtaining one unit of content when $N$ peers share all produced content. Thus, at PE, the level of total production is chosen to equate the marginal benefit and the marginal cost of supplying content to every peer in the P2P network.

Remark. Proposition 2 determines production up to the aggregate level, leaving the individual levels unspecified. This is a by-product of the linear cost function of production (i.e., constant returns to scale). If we assume a strictly convex cost function (i.e., decreasing returns to scale), $c\left(x_{i}\right)$, instead of $\kappa x_{i}$, then PE requires that every peer produce the same amount $x^{o}$ that maximizes $f(N x)-c(x)-(N-1)(\delta+\sigma) x$, eliminating indeterminacy in the allocation of total production to peers.

At PE, peers jointly produce $\hat{x}_{\beta}$ and share all produced content so that each peer consumes the total amount produced. The utility of peer $i$ producing $x_{i}^{o}$ at a PE allocation $\left(\mathbf{x}^{o}, \mathbf{y}^{o}, \mathbf{Z}^{o}\right)$ is given by

$$
v_{i}\left(\mathbf{x}^{o}, \mathbf{y}^{o}, \mathbf{Z}^{o}\right)=f\left(\hat{x}_{\beta}\right)-\delta \hat{x}_{\beta}-[\kappa+(N-1) \sigma-\delta] x_{i}^{o} .
$$

Note that the utility of a peer is decreasing in its production level given that the total amount of production is fixed and that all produced content is shared. Total utility at $\mathrm{PE}$ is given by $\Pi^{P E}=N f^{*}(\beta)$. Since $\beta<\kappa$ for $N \geq 2$, the consumption of a peer and total utility are smaller at non-cooperative equilibrium than at PE, i.e., $\hat{x}_{\kappa}<\hat{x}_{\beta}$ and $\Pi^{N C}<\Pi^{P E}$.

In order to derive a coalitional game [27] based on the CPS game, we need to compute the maximum total utility that a subset of peers can achieve. Define $\tilde{\beta}(n)$ by

$$
\tilde{\beta}(n)=\frac{1}{n} \kappa+\frac{n-1}{n}(\delta+\sigma)
$$

for $n=1,2, \ldots$. Note that $\tilde{\beta}(1)=\kappa, \tilde{\beta}(N)=\beta$, and $\tilde{\beta}(n) \rightarrow \delta+\sigma$ as $n \rightarrow \infty$. $\tilde{\beta}(n)$ can be interpreted as the per capita marginal cost of obtaining one unit of content when $n$ peers share all produced content. The maximum total utility achievable with $n$ peers is given by $G(n) \triangleq n f^{*}(\tilde{\beta}(n))$, and the maximum average individual utility achievable with $n$ peers by $g(n) \triangleq G(n) / n=f^{*}(\tilde{\beta}(n))$. Marginal product (MP) measures an increment in the maximum total utility when the $n$th peer joins the P2P network, i.e., $M P(1) \triangleq G(1)$ and $M P(n) \triangleq G(n)-G(n-1)=n f^{*}(\tilde{\beta}(n))-(n-1) f^{*}(\tilde{\beta}(n-1))$ for $n \geq 2$. The following proposition gives some properties of the functions $g$ and $M P$. 
Proposition 3. (i) $g(n)$ is increasing in $n$, and $\lim _{n \rightarrow \infty} g(n)=f^{*}(\delta+\sigma)$.

(ii) $M P(n)$ is increasing in $n, M P(n)>g(n)$ for all $n \geq 2$, and $\lim _{n \rightarrow \infty}[M P(n)-g(n)]=0$.

Since $g(n)$ is increasing in $n$, there are increasing returns to scale when inputs and outputs are taken to be peers and total utility, respectively. As there are more peers in the P2P network, the cost of production can be shared by more peers and the socially valuable P2P network can be utilized more extensively, which results in an increase in the maximum average individual utility 10 Proposition 3(i) in addition states that the maximum average individual utility is bounded above. Proposition 3(ii) shows that the no-surplus condition in the sense of [29] is satisfied only in the limiting case with infinitely many peers. This implies that the distribution of total utility to peers according to their MP, which is proposed by the marginal productivity theory of distribution of neoclassical economics, is not feasible unless there are infinitely many peers. Thus, we rely on cooperative game theory as an alternative theory of distribution.

Let $\mathcal{S}$ with $\mathcal{S} \neq \emptyset$ and $\mathcal{S} \subseteq \mathcal{N}$ be a coalition of peers. The characteristic function $v$, which assigns each coalition the maximum total utility it can create, is given by

$$
v(\mathcal{S})=|\mathcal{S}| f^{*}(\tilde{\beta}(|\mathcal{S}|)),
$$

where $|\mathcal{S}|$ denotes the number of peers in coalition $\mathcal{S}$. We set $v(\emptyset)=0$. A coalitional game is described by the characteristic function $v$, and we consider two solution concepts for coalitional games, the core and the Shapley value. An allocation $(\mathbf{x}, \mathbf{y}, \mathbf{Z})$ has the core property in the coalitional game $v$ if

$$
\sum_{i \in \mathcal{N}} v_{i}(\mathbf{x}, \mathbf{y}, \mathbf{Z})=v(\mathcal{N}) \text { and } \sum_{i \in \mathcal{S}} v_{i}(\mathbf{x}, \mathbf{y}, \mathbf{Z}) \geq v(\mathcal{S}), \forall \mathcal{S} \subseteq \mathcal{N} .
$$

The first condition states that the maximum total utility with the grand coalition $\mathcal{N}$ is distributed to peers (i.e., a PE allocation is chosen) while the second condition states that no coalition can improve the utilities of its members from the current allocation. Hence, the core describes the stable distributions of total utility in that no coalition of peers can improve their utilities by separating from the grand coalition. This implies that, when the protocol designer enforces an allocation with the core property, no coalition of peers can object the allocation credibly by threatening to leave the P2P network. The Shapley value, whose expression can be found in [27], is a distribution of total utility, $v(\mathcal{N})$, that satisfies a certain set

\footnotetext{
${ }^{10}$ In our model, there are no congestion effects in that the marginal costs of upload and download are independent of the number of peers in the network. If we generalize our model so that the marginal costs of upload and download are increasing in the number of peers, then additional peers will have not only positive externalities but also negative externalities on the existing peers and there may exist an optimal network size that maximizes total utility as in [28].
} 


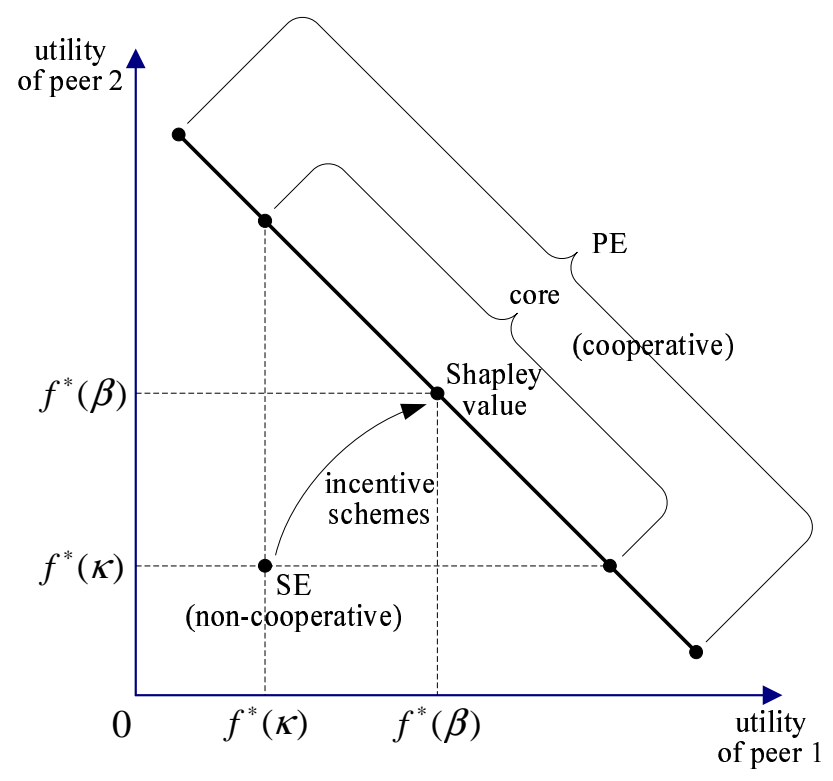

Fig. 1: Two-peer illustration of utility profiles achieved at non-cooperative and cooperative solution concepts. Incentive schemes allow non-cooperative peers to achieve a cooperative outcome.

of axioms. The Shapley value can be considered as a fair distribution of utility as it takes into account the MP of peers in all possible orders of arrival in the P2P network.

Proposition 4. (i) The core of the coalitional game $v$ is a nonempty convex set whose vertices are given by $(M P(1), M P(2), \ldots, M P(N))$ and all of its permutations. At the core, we have $\sum_{i=1}^{N} x_{i}=\hat{x}_{\beta}$, $x_{i}=y_{i}=z_{j i}$ for all $j \neq i$, for all $i \in \mathcal{N}$, and

$$
\sum_{i \in \mathcal{S}} x_{i} \leq|\mathcal{S}| \frac{f\left(\hat{x}_{\beta}\right)-\delta \hat{x}_{\beta}-f^{*}(\tilde{\beta}(|\mathcal{S}|))}{\kappa+(N-1) \sigma-\delta}
$$

for all $\mathcal{S} \subseteq \mathcal{N}$.

(ii) The Shapley value of the coalitional game $v$ is $v_{i}=f^{*}(\beta)$ for all $i \in \mathcal{N}$, which is attained at the symmetric PE allocation, $x_{i}=y_{i}=z_{j i}=\hat{x}_{\beta} / N$ for all $j \neq i$, for all $i \in \mathcal{N}$.

Fig. 1 illustrates the results in Propositions 1, 2, and 4 with two peers. Since $\Pi^{N C}<\Pi^{P E}$, PE allocations achieve a higher total utility than SE allocations. Also, since the core imposes additional constraints on PE, the core is a subset of PE utility profiles. Proposition 3(ii) implies that the coalitional game $v$ is convex [30], and thus the results in Proposition 4 can be considered as the corollaries of theorems in [30]. In particular, the core is nonempty and coincides with the unique stable set in the sense of [31]. Also, the Shapley value is the center of gravity of the core, which is consistent with the 
illustration in Fig. 11 Hence, by prescribing the allocation that yields the Shapley value, the protocol designer can obtain the stability property of the core and the fairness property of the Shapley value at the same time.

The maximum utility that a peer can obtain by itself is $f^{*}(\kappa)$, which can be considered as a reservation utility. An allocation $(\mathbf{x}, \mathbf{y}, \mathbf{Z})$ satisfies the participation (or individual rationality) constraint for peer $i$ if $v_{i}(\mathbf{x}, \mathbf{y}, \mathbf{Z}) \geq f^{*}(\kappa)$. An allocation $(\mathbf{x}, \mathbf{y}, \mathbf{Z})$ is participation-efficient if it is $\mathrm{PE}$ and satisfies the participation constraint for every peer. Among PE allocations, condition (3) for a singleton coalition $\mathcal{S}=\{i\}$, which can be written as

$$
x_{i} \leq \frac{f\left(\hat{x}_{\beta}\right)-\delta \hat{x}_{\beta}-f^{*}(\kappa)}{\kappa+(N-1) \sigma-\delta},
$$

is required for the participation constraint for peer $i$. Since the utility of a peer decreases in its production level among PE allocations as shown in (1), the participation constraint puts an upper bound on the individual production level to prevent a peer from leaving the $\mathrm{P} 2 \mathrm{P}$ network. The core is a stronger concept than participation-efficiency in that the core prevents not only a single peer from leaving the P2P network but also a subset of peers from forming their own P2P network.

Peers choose actions $\mathbf{x}, \mathbf{y}$, and $\mathbf{Z}$ over the three stages of the CPS game. Suppose that the protocol designer can enforce the sharing levels of peers in stage two while he cannot enforce the choices in stages one and three. Then the CPS game is reduced to the CPS game with enforced sharing levels $\mathbf{y}^{e}$, where the stage-two choice of peers is fixed at some $\mathbf{y}^{e}$.

Proposition 5. Suppose that $\hat{x}_{\kappa} \leq \sum_{i=1}^{N} y_{i}^{e} \leq \hat{x}_{\delta}$. At the SE outcome of the CPS game with enforced sharing levels $\mathbf{y}^{e}$, we have $x_{i}=y_{i}^{e}=z_{j i}$ for all $j \neq i$, for all $i \in \mathcal{N}$.

Proposition 5 shows that when peers are required to share $\mathbf{y}^{e}$ that satisfies $\hat{x}_{\kappa} \leq \sum_{i=1}^{N} y_{i}^{e} \leq \hat{x}_{\delta}$, they produce exactly the enforced sharing levels and download all shared content in their self-interest. Since $\hat{x}_{\kappa} \leq \hat{x}_{\beta} \leq \hat{x}_{\delta}$, the protocol designer can implement a PE allocation by enforcing only the sharing levels $\mathbf{y}^{e}$ such that $\sum_{i=1}^{N} y_{i}^{e}=\hat{x}_{\beta}$, leaving peers to choose the production and download levels on their own.

\section{PAYMENT SCHEMES}

Suppose that the protocol designer is unable to enforce the sharing levels of peers. The non-cooperative analysis in Section III suggests that, in order to avoid the collapse of the P2P network, peers need to be incentivized to share and upload their produced content. Pricing is an extensively studied form of incentives to achieve an efficient use of network resources [32]. Pricing schemes have been used in 
P2P-based web services such as MojoNation in the forms of tokens and credits. We say that a pricing scheme is optimal if it induces a PE non-cooperative equilibrium. In order to determine an optimal pricing scheme, the protocol designer needs to know the utility functions of peers. An optimal pricing scheme can be implemented when the protocol designer can enforce payments and each peer knows the pricing rule applied to it.

In this section, we examine a class of pricing schemes under which the payment to a peer is increasing in its upload volume and decreasing in its download volume at the same rate 11 We call such a pricing scheme a linear pricing scheme, which can be expressed formally as

$$
t_{i}(\mathbf{Z})=p\left(u_{i}-d_{i}\right)
$$

for some price $p>0$. Note that a linear pricing scheme with any price satisfies budget balance since it simply transfers payments from downloaders to uploaders, i.e., $\sum_{i=1}^{N} t_{i}(\mathbf{Z})=p\left(\sum_{i=1}^{N} u_{i}-\sum_{i=1}^{N} d_{i}\right)=0$ for all $\mathbf{Z}$ and $p$. The payoff to peer $i$ in the CPS game with the linear pricing scheme with price $p$ is given by

$$
\pi_{i}(\mathbf{x}, \mathbf{y}, \mathbf{Z})=v_{i}(\mathbf{x}, \mathbf{y}, \mathbf{Z})+t_{i}(\mathbf{Z})=f\left(x_{i}+d_{i}\right)-\kappa x_{i}-(p+\delta) d_{i}+(p-\sigma) u_{i}
$$

In effect, the linear pricing scheme with price $p$ increases the cost of download from $\delta$ to $p+\delta$ and decreases the cost of upload from $\sigma$ to $\sigma-p$. If the reward for upload exceeds the cost of upload, i.e., $p>\sigma$, then peers receive a net benefit from uploading, which provides them with an incentive for sharing. The following proposition shows that there exists an optimal pricing scheme in the class of linear pricing schemes. Moreover, the optimal linear pricing scheme transfers the utilities of peers so that the equilibrium payoff profile coincides with the Shapley value for any PE allocation chosen by peers.

Proposition 6. Let $p^{*}=[\kappa+(N-1) \sigma-\delta] / N$. At the SE outcome of the CPS game with the linear pricing scheme with price $p^{*}$, we have $\sum_{i=1}^{N} x_{i}=\hat{x}_{\beta}$ and $x_{i}=y_{i}=z_{j i}$ for all $j \neq i$, for all $i \in \mathcal{N}$. The payoff of each peer at $S E$ is given by $f^{*}(\beta)$.

Proof: The result follows from Lemma 1 and Proposition 7 of [20].

Remark. As is the case with PE, indeterminacy in the allocation of total production to peers can be eliminated by assuming a strictly convex cost function of production, $c\left(x_{i}\right)$. With a strictly convex cost

\footnotetext{
${ }^{11}$ Another class of pricing schemes, called MP pricing schemes, is proposed and analyzed in [20]. Under an MP pricing scheme, payments are determined based on the sharing levels of peers.
} 
function of production, the optimal price $p^{*}$ can be found as in Proposition 6 replacing $\kappa$ with $c^{\prime}\left(x^{o}\right)$, where $x^{o}$ is the individual production level at PE as discussed in the remark following Proposition 2.

A notable feature of the optimal price $p^{*}$ is that peers are indifferent between the two alternative methods of obtaining data, production and download, when they face the optimal price. At PE without pricing schemes, peers prefer download to production because the marginal cost of download, $\delta$, is smaller than that of production and upload, $\kappa+(N-1) \sigma$ (see the coefficient of the term $x_{i}^{o}$ in (1)). The optimal price is chosen such that it equates the effective marginal cost of download, $p^{*}+\delta$, with that of production and upload, $\kappa-(N-1)\left(p^{*}-\sigma\right)$. As a result, at SE with the optimal linear pricing scheme, peers obtain the same payoff regardless of their production levels.

With linear cost functions and linear prices, this is a necessary property of any nondiscriminatory pricing scheme that induces non-cooperative homogeneous peers to produce a positive bounded amount of content in aggregate. If the payoff to a peer is increasing in its production level, then the peer would be willing to produce and upload as much as it can (i.e., the peer is overcompensated for its production and the supply of content is unbounded). On the other hand, if the payoff to a peer is decreasing in its production level, then the peer would not produce at all (i.e., the peer is undercompensated for its production and the supply of content is zero). The optimal price can be considered as an equilibrium price of content in that the compensation is enough to provide incentives for production and upload but prevents overproduction so that supply equals demand.

Imposing that $y_{i}=x_{i}$ and $u_{i}=(N-1) y_{i}$ for all $i \in \mathcal{N}$, the social welfare maximization problem can be written as

$$
\begin{aligned}
& \max _{\mathbf{x}, \mathbf{d}} \sum_{i=1}^{N}\left\{f\left(x_{i}+d_{i}\right)-[\kappa+(N-1) \sigma] x_{i}-\delta d_{i}\right\} \\
& \text { subject to } x_{i} \geq 0,0 \leq d_{i} \leq \sum_{j \neq i} x_{j}, \text { for all } i=1, \ldots, N .
\end{aligned}
$$

Let $p_{i} \geq 0$ be a Lagrange multiplier on the constraint $d_{i} \leq \sum_{j \neq i} x_{j}$ for each $i \in \mathcal{N}$, which can be interpreted as the price that peer $i$ pays for its download. Then the Lagrangian function can be written as

$$
\mathcal{L}(\mathbf{x}, \mathbf{d} ; \mathbf{p})=\sum_{i=1}^{N}\left\{f\left(x_{i}+d_{i}\right)-[\kappa+(N-1) \sigma] x_{i}-\delta d_{i}+p_{i}\left(\sum_{j \neq i} x_{j}-d_{i}\right)\right\},
$$

and the first-order optimality conditions for $x_{i}$ and $d_{i}$ are given by

$$
f^{\prime}\left(x_{i}+d_{i}\right)-\kappa-(N-1) \sigma+\sum_{j \neq i} p_{j} \leq 0 \text { (with equality if } x_{i}>0 \text { ) }
$$


and

$$
\left.f^{\prime}\left(x_{i}+d_{i}\right)-\delta-p_{i} \leq 0 \text { (with equality if } d_{i}>0\right) \text {. }
$$

At PE, we have $x_{i}+d_{i}=\hat{x}_{\beta}$, which yields the Lagrange multiplier $p_{i}=p^{*}$ for all $i \in \mathcal{N}$. The dual decomposition of (4) can be written as

$$
\max _{x_{i}, d_{i} \geq 0} f\left(x_{i}+d_{i}\right)-\left[\kappa+(N-1) \sigma-\sum_{j \neq i} p_{j}\right] x_{i}-\left(p_{i}+\delta\right) d_{i}
$$

for each $i$. The solution to (5) is given by

$$
\begin{array}{ll}
x_{i}=0 \text { and } d_{i}=\hat{x}_{\left(p_{i}+\delta\right)} & \text { if } \sum_{j=1}^{N} p_{j}<\kappa+(N-1) \sigma-\delta, \\
x_{i}+d_{i}=\hat{x}_{\left(p_{i}+\delta\right)}=\hat{x}_{\left[\kappa+(N-1) \sigma-\sum_{j \neq i} p_{j}\right]} & \text { if } \sum_{j=1}^{N} p_{j}=\kappa+(N-1) \sigma-\delta, \\
x_{i}=\hat{x}_{\left[\kappa+(N-1) \sigma-\sum_{j \neq i} p_{j}\right]} \text { and } d_{i}=0 & \text { if } \sum_{j=1}^{N} p_{j}>\kappa+(N-1) \sigma-\delta
\end{array}
$$

Thus, the maximum value of (5) is given by $h_{i}(\mathbf{p}) \triangleq f^{*}\left(\min \left\{p_{i}+\delta, \kappa+(N-1) \sigma-\sum_{j \neq i} p_{j}\right\}\right)$, and $p_{i}=p^{*}$ for all $i$ is the solution of the dual problem, $\min _{\mathbf{p} \geq 0} \sum_{i=1}^{N} h_{i}(\mathbf{p})$. Thus, a uniform linear pricing scheme suffices to obtain PE allocations. The problem (4) is more general than the resource allocation problem in [33] in the following aspect. In our problem, peers can choose to become either a seller or a buyer (or both), and the amount of resources (i.e., content) supplied in the P2P network is chosen by peers. On the contrary, in [33], buyers and sellers are predetermined, and sellers hold a fixed supply of resources.

If the protocol designer knows the utility functions of peers, he can compute the optimal price $p^{*}$ using the expression in Proposition 6. At the optimal price, equilibrium requires that peers produce $\hat{x}_{\beta}$ in aggregate. Peers can coordinate to achieve total production $\hat{x}_{\beta}$ using the following quantity adjustment process. Initially, each peer $i$ chooses arbitrary optimal production and download levels $\left(x_{i}, d_{i}\right)$, which satisfy $x_{i}+d_{i}=\hat{x}_{\beta}$. Peers share their production fully throughout the process, and thus they can observe the production levels of other peers indirectly. If $\sum_{i=1}^{N} x_{i}>\hat{x}_{\beta}$ (respectively, $\sum_{i=1}^{N} x_{i}<\hat{x}_{\beta}$ ), then $d_{i}<\sum_{j \neq i} x_{j}$ (respectively, $d_{i}>\sum_{j \neq i} x_{j}$ ) for all $i$. Hence, if each peer $i$ adjusts its production and download levels by

$$
\frac{d x_{i}}{d t}=-\frac{d d_{i}}{d t}=\eta_{i}\left(d_{i}-\sum_{j \neq i} x_{j}\right)
$$

\footnotetext{
${ }^{12}$ We set $\hat{x}_{\alpha}=+\infty$ when $\alpha \leq 0$.
} 
for some constant $\eta_{i}>0.13$ then the allocation will converge to an equilibrium allocation, which satisfies $\sum_{i=1}^{N} x_{i}=\hat{x}_{\beta}$.

Suppose instead that the protocol designer does not know the utility functions of peers. In this case, the protocol designer can still find the optimal price by using a price adjustment process similar to that in [34]. In other words, a price adjustment process can substitute knowledge about the utility functions of peers. In the proposed price adjustment process, the protocol designer announces a price $p$, which applies to every peer. Given the price, each peer $i$ chooses $\left(x_{i}, d_{i}\right)$ by solving (5) and reports its choice to the protocol designer. We assume that peers can coordinate their choices to satisfy $d_{i}=\sum_{j \neq i} x_{j}$ for all $i \in \mathcal{N}$ whenever possible, for example, by using the quantity adjustment process (7). The total demand for content at price $p$ is denoted by $D(p)$ and can be computed as $\sum_{i=1}^{N} d_{i}$. Similarly, the total supply of content at price $p$ is denoted by $S(p)$ and can be computed as $\sum_{i=1}^{N} u_{i}=(N-1) \sum_{i=1}^{N} x_{i}$, as a peer can upload its production up to $(N-1)$ times. Using (6), we obtain

$$
D(p)=\left\{\begin{array}{ll}
N \hat{x}_{(p+\delta)} & \text { if } p<p^{*} \\
(N-1) \hat{x}_{\beta} & \text { if } p=p^{*} \\
0 & \text { if } p>p^{*}
\end{array} \text { and } \quad S(p)= \begin{cases}0 & \text { if } p<p^{*} \\
(N-1) \hat{x}_{\beta} & \text { if } p=p^{*} \\
N(N-1) \hat{x}_{[\kappa-(N-1)(p-\sigma)]} & \text { if } p>p^{*}\end{cases}\right.
$$

as depicted in Fig. 2. We define the excess demand at price $p$ by $E D(p) \triangleq D(p)-S(p)$. The protocol designer adjusts the price following the process

$$
\frac{d p}{d t}=\eta E D(p)
$$

for some constant $\eta>0$. Since $E D(p)>0$ for $p<p^{*}$ and $E D(p)<0$ for $p>p^{*}$, the price will converge to the optimal price $p^{*}$ starting from any initial price. We compare the above price adjustment process with that in [34]. In [34], there are multiple resources with fixed supply, and each resource manager adjusts the price of his resource so that aggregate demand for the resource equals the supply of the resource. In our formulation, by focusing on a uniform linear pricing scheme, we treat resources provided by different peers as a single resource. Hence, the protocol designer needs to aggregate demand and supply by all peers and adjust the price of content to eliminate excess demand or supply.

We have assumed that peers report their demand and supply truthfully in the price adjustment process. Suppose instead that peers know the price adjustment process used by the protocol designer and can engage in strategic misrepresentation as in [35]. We find that, unlike in [35], no peer can gain from influencing the equilibrium price by misreporting its demand or supply provided that other peers report

\footnotetext{
${ }^{13}$ Since $x_{i}$ cannot be negative, we assume that peer $i$ stops adjusting its quantity when $x_{i}=0$ and $d_{i}<\sum_{j \neq i} x_{j}$.
} 


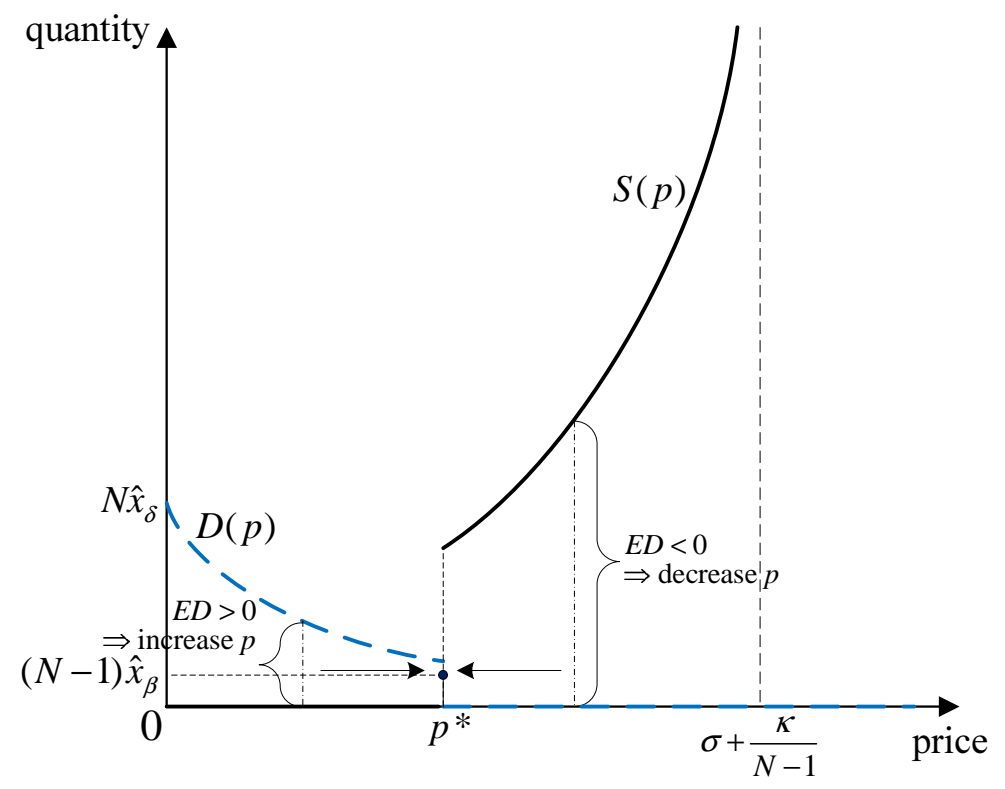

Fig. 2: Equilibrium interpretation of the optimal price $p^{*} . D(p)$ represents the total demand for download and $S(p)$ the total supply of upload at price $p$. The equilibrium price equates demand and supply, i.e., $D\left(p^{*}\right)=S\left(p^{*}\right)$. The protocol designer can reach the equilibrium price by adjusting the price depending on the excess demand, ED.

truthfully. In [35], a user, acting as a buyer, can benefit from a lower price of a resource by underreporting its demand. On the contrary, in our model, a peer is both a buyer and a seller, and thus it can lower the price only by increasing its supply, which hurts it as a seller. Peer $i$ can make the price adjustment process stop at $p^{\prime}<p^{*}$ by reporting $x_{i}=\hat{x}_{\left(p^{\prime}+\delta\right)}$ and $d_{i}=0$. Since $p^{\prime}+\delta<\beta<\kappa-(N-1)\left(p^{\prime}-\sigma\right)$, the payoff of peer $i$ is

$$
\pi_{i}=f\left(\hat{x}_{\left(p^{\prime}+\delta\right)}\right)-\left[\kappa-(N-1)\left(p^{\prime}-\sigma\right)\right] \hat{x}_{\left(p^{\prime}+\delta\right)}<f\left(\hat{x}_{\left(p^{\prime}+\delta\right)}\right)-\beta \hat{x}_{\left(p^{\prime}+\delta\right)}<f^{*}(\beta),
$$

and thus it obtains a lower payoff by manipulating the equilibrium price at $p^{\prime}$. For $p>p^{*}$, the optimal production and download levels for peer $j$ are $x_{j}=\hat{x}_{[\kappa-(N-1)(p-\sigma)]}$ and $d_{j}=0$. Since $d_{i} \leq \sum_{j \neq i} x_{j}$, peer $i$ alone cannot induce $E D(p)=0$, or $\sum_{i=1}^{N} d_{i}=(N-1) \sum_{i=1}^{N} x_{i}$, for some $p>p^{*}$, if $N>2$. When $N=2$, peer $i$ can make the price adjustment process stop at $p^{\prime \prime}>p^{*}$ by reporting $x_{i}=0$ and $d_{i}=\hat{x}_{\left[\kappa-(N-1)\left(p^{\prime \prime}-\sigma\right)\right]}$. Since $\kappa-(N-1)\left(p^{\prime \prime}-\sigma\right)<\beta<p^{\prime \prime}+\delta$, the payoff of peer $i$ is

$$
\begin{aligned}
\pi_{i} & =f\left(\hat{x}_{\left[\kappa-(N-1)\left(p^{\prime \prime}-\sigma\right)\right]}\right)-\left(p^{\prime \prime}+\delta\right) \hat{x}_{\left[\kappa-(N-1)\left(p^{\prime \prime}-\sigma\right)\right]} \\
& <f\left(\hat{x}_{\left[\kappa-(N-1)\left(p^{\prime \prime}-\sigma\right)\right]}\right)-\beta \hat{x}_{\left[\kappa-(N-1)\left(p^{\prime \prime}-\sigma\right)\right]}<f^{*}(\beta) .
\end{aligned}
$$


Again, peer $i$ cannot gain from misreporting.

\section{Differential Service Schemes}

Another form of incentives that encourage sharing by non-cooperative peers is differential service, in which peers obtain different qualities of service depending on their contribution levels. Differential service schemes are widely adopted in file sharing P2P networks such as BitTorrent [36] and KaZaA, in the forms of tit-for-tat and reputation. In this section, we capture the differential service in the CPS game using two modeling approaches based on repeated games and intervention. In a repeated game model, peers can reciprocate service to each other based on private or public history. In an intervention model, the system treats peers differentially based on their contribution to the system.

\section{A. Repeated Game Model}

Suppose that peers interact repeatedly over time in the P2P network. The repeated game model can support a cooperative outcome among non-cooperative peers by providing rewards and punishments depending on the past behavior of peers. The repeated CPS game is a supergame in which the CPS game is played repeatedly. We use the limit of means criterion [37] to evaluate the utility of a peer in the repeated CPS game to obtain the following result 14

Proposition 7. Any participation-efficient allocation can be supported as a non-cooperative equilibrium of the repeated CPS game.

By Proposition 5, any deviation that is profitable in the current CPS game involves a deviation in sharing levels, which can be publicly observed by peers. Hence, the protocol designer can deter peers from free-riding in the P2P network by making peers play the SE of the one-shot CPS game in all subsequent CPS games whenever a peer does not share its required amount of content. We have assumed that peers serve all the download requests they receive in stage three. Suppose instead that a peer can choose whether to upload or not to another peer that requests its content. Then the punishment following a deviation in sharing levels can be asymmetric by prescribing peers not to upload to a peer that has ever deviated, which effectively excludes the deviating peer from the P2P network. Similarly, refusing a download request from a peer that has not deviated can also be deterred by using private retaliation

\footnotetext{
${ }^{14} \mathrm{~A}$ similar result can be obtained with the discounting criterion, in which case Proposition 7 is restated as "Any strictly participation-efficient allocation can be supported as a non-cooperative equilibrium of the repeated CPS game when peers are sufficiently patient."
} 
(i.e., a non-deviating peer whose request was refused does the same thing in return to the peer that has refused its request) in all subsequent CPS games.

\section{B. Intervention Model}

Intervention [38] refers to the system directly influencing the usage of users depending on their behavior. We consider a particular form of intervention applicable to P2P networks. Suppose that the P2P network can reduce the download rate of a peer depending on its rating, where the rating of peer $i$ is defined by its upload to download ratio, i.e., $r_{i}=u_{i} / d_{i}$. Then a differential service scheme based on intervention can be described by an intervention function $q: \mathbb{R}_{+} \rightarrow \mathbb{R}_{+}$, which represents an increase in the marginal cost of download. That is, when peer $i$ has rating $r_{i}$, its marginal cost of download after intervention is given by $\delta+q\left(r_{i}\right)$. Note that the range of $q$ is constrained to be nonnegative since we assume that the system can only decrease the download rates. This imposes a restriction in incentive design compared to a payment scheme, where it is usually assumed that a payment function can take any positive or negative real number. However, incentive schemes based on intervention have advantages in implementation over those based on payment and repeated games. Unlike a payment scheme, there is no need for transactions in an intervention scheme since intervention affects peers directly through the system. Also, intervention can be considered as a substitute of the punishment strategy in repeated games, but it requires neither repeated interaction among peers nor the maintenance of history since punishment is executed by the system architecture rather than by peers 15 We say that an intervention scheme is optimal if it achieves a PE allocation with zero intervention level at non-cooperative equilibrium. Since any positive level of intervention results in performance degradation, it is desirable to have intervention only as a threat, which is called for when misbehavior occurs.

Proposition 8. Define an intervention function $q^{*}$ by $q^{*}\left(r_{i}\right)=p^{*}\left[1-r_{i}\right]^{+}$, where $p^{*}=[\kappa+(N-1) \sigma-\delta] / N$ and $[r]^{+}=\max \{r, 0\}$. At the SE outcome of the CPS game with the intervention scheme $q^{*}$, we have $x_{i}=y_{i}=z_{j i}=\hat{x}_{\beta} / N$ for all $j \neq i$, for all $i \in \mathcal{N}$. Moreover, $q^{*}\left(r_{i}\right)=0$ at $S E$.

Proof: As long as $r_{i} \leq 1$, or $u_{i} \leq d_{i}$, for all $i$, the intervention scheme $q^{*}$ is equivalent to the optimal linear pricing scheme $p^{*}$. Since increasing $u_{i}$ beyond $d_{i}$ can only increase the cost of upload without affecting the level of intervention, we must have $u_{i} \leq d_{i}$ for all $i$ at SE. Among SE with the

\footnotetext{
${ }^{15}$ For example, the considered type of intervention can be implemented in a distributed way by requiring peers to use a certain program to download and upload files, which can adjust the download rate of a peer automatically based on its past usage.
} 
optimal pricing scheme $p^{*}$ given in Proposition $6, u_{i} \leq d_{i}$ is satisfied for all $i$ only when the amount of total production $\hat{x}_{\beta}$ is split equally to all peers. At this allocation, $u_{i}=d_{i}=(N-1) \hat{x}_{\beta} / N$, and thus $r_{i}=1$ and $q^{*}\left(r_{i}\right)=0$ at SE.

Proposition 8 shows that an optimal intervention scheme can be constructed to achieve the symmetric PE allocation without intervening at equilibrium. Under the optimal intervention scheme $q^{*}$, a peer experiences a reduced download rate whenever it downloads more than it uploads. Since every peer downloads and uploads the same amount at the symmetric PE allocation, reduced download rates act only as a threat at equilibrium, deterring peers from deviation. The model of [7] can be considered as using another form of intervention, where the system determines the proportion of shared content that a peer is allowed to download as a function of the contribution of the peer. The model of [22] can also be interpreted as using an intervention scheme, where the system no longer serves a peer when its cumulative average rating falls below a threshold level. [38] applies an intervention scheme to a multi-user access network, where the system can jam packets randomly with a probability that depends on the transmission probabilities of users. In [38], intervention affects all users in the system to the same degree, thus represented by a function that depends on the actions of all users. On the contrary, intervention considered in the CPS game, [7], and [22] influences a peer depending only on its own action, thus allowing the differential service to peers.

\section{ENFORCED FULL SHARING}

We have seen from Proposition 5 that the protocol designer can achieve a PE allocation by enforcing the sharing levels of peers. As an alternative scenario, suppose that the protocol designer can enforce full sharing among peers, but not sharing levels 16 The resulting CPS game is called the CPS game with enforced full sharing. Formally, the CPS game with enforced full sharing is a restricted version of the CPS game where the stage-two choice of each peer $i$ is fixed as $y_{i}=x_{i}$. Note that enforced sharing levels constrain the production decisions of peers in that peers need to produce at least the required sharing levels. On the contrary, under enforced full sharing, peers can choose any levels of production in stage one. The following proposition characterizes allocations at the SE of the CPS game with enforced full sharing.

Proposition 9. Let $\gamma \triangleq \kappa+(N-1) \sigma$. Define $\tilde{x}_{\gamma}$ by $\tilde{x}_{\gamma}=\hat{x}_{\gamma}$ if $\gamma \leq f^{\prime}(0)$ and $\tilde{x}_{\gamma}=0$ otherwise (i.e.,

\footnotetext{
${ }^{16}$ For example, full sharing can be enforced when there exists an indispensable technology for production and peers have access to it under the condition of sharing the produced content.
} 
$\left.\tilde{x}_{\gamma}=\arg \max _{x \geq 0}\{f(x)-\gamma x\}\right)$. At the SE outcome of the CPS game with enforced full sharing, we have $\sum_{i=1}^{N} x_{i}=\tilde{x}_{\gamma}$ and $x_{i}=y_{i}=z_{j i}$ for all $j \neq i$, for all $i \in \mathcal{N}$. Thus, $w(\mathbf{Z})=(N-1) \tilde{x}_{\gamma}$ at SE with enforced full sharing.

Proof: A formal proof can be found in [20, Prop. 3]. Since peers download all shared content at $\mathrm{SE}$, enforced full sharing increases the effective marginal cost of production from $\kappa$ to $\kappa+(N-1) \sigma$, which includes the marginal cost of upload to $(N-1)$ peers. The stage-one problem for peer $i$ can be written as

$$
\max _{x_{i} \geq 0} f\left(\sum_{i=1}^{N} x_{i}\right)-[\kappa+(N-1) \sigma] x_{i}-\delta \sum_{j \neq i} x_{j}
$$

given $\mathbf{x}_{-i}$, and the result follows.

As peers face effectively a higher cost of production with enforced full sharing, non-cooperative peers reduce their production when full sharing is enforced, i.e., $\tilde{x}_{\gamma}<N \hat{x}_{\kappa}$. Total utility at SE with enforced full sharing is given by $\Pi^{F S}=N\left[f\left(\tilde{x}_{\gamma}\right)-\beta \tilde{x}_{\gamma}\right]$.

To make welfare comparisons, we first consider a scenario in which the number of peers in the P2P network is fixed as $N$. The price of anarchy (PoA) 17 is defined to be the ratio of social welfare at the worst non-cooperative equilibrium to that at PE, i.e.,

$$
P o A \triangleq \frac{\Pi^{N C}}{\Pi^{P E}}=\frac{f^{*}(\kappa)}{f^{*}(\beta)} .
$$

The price of no sharing (PoNS) compares social welfare at SE with and without enforced full sharing, i.e.,

$$
P o N S \triangleq \frac{\Pi^{N C}}{\Pi^{F S}}=\frac{f^{*}(\kappa)}{f\left(\tilde{x}_{\gamma}\right)-\beta \tilde{x}_{\gamma}}\left(=+\infty \text { if } \tilde{x}_{\gamma}=0\right) .
$$

Finally, the price of underproduction (PoU) compares social welfare at SE with enforced full sharing and at PE, i.e.,

$$
P o U \triangleq \frac{\Pi^{F S}}{\Pi^{P E}}=\frac{f\left(\tilde{x}_{\gamma}\right)-\beta \tilde{x}_{\gamma}}{f^{*}(\beta)} .
$$

When $\tilde{x}_{\gamma}>0$, the PoA can be decomposed as the product of the PoNS and the PoU, i.e., PoA= $P o N S \times P o U$. The PoA is a widely used measure of the inefficiency of non-cooperative equilibria. The PoNS measures the welfare implication of enforced full sharing on selfish peers, and thus it can be used to analyze the value of a technology that enables enforced full sharing. The PoU measures inefficiency

\footnotetext{
${ }^{17}$ Since the non-cooperative outcome of the CPS game is unique, the price of anarchy and the price of stability coincide for the CPS game.
} 
due to underproduction caused by the selfish behavior of peers assuming that full sharing is enforced. The following proposition examines the range of values that each measure of inefficiency can take when we vary the utility specification of the model, $f, \kappa, \delta$, and $\sigma$.

Proposition 10. For a fixed size $N \geq 2$ of the P2P network, $\operatorname{Po} A \in(0,1), \operatorname{PoN} S \in(0, \infty]$, and PoU $\in[0,1)$. These bounds are tight.

Proof: A formal proof can be found in [20, Prop. 4].

Since $\beta<\kappa<\gamma$ for $N \geq 2$, it follows immediately from (8) and (9) that $\operatorname{PoA}, \operatorname{PoU}<1$, which shows that selfish behavior results in efficiency losses regardless of whether full sharing is enforced or not. The relative size of $\Pi^{N C}$ and $\Pi^{F S}$ is ambiguous, which implies that the enforcement of full sharing may make peers worse off. This is because enforced full sharing has two offsetting effects on social welfare. On one hand, full sharing has a positive effect on welfare by reducing the cost of obtaining one unit of content to $\beta$, compared to $\kappa$ in the case of no sharing. On the other hand, full sharing has a negative effect by increasing the effective cost of producing one unit of content from $\kappa$ to $\gamma$. Therefore, the overall welfare implication of enforced full sharing is determined by the stronger of the two effects.

Next we consider a scenario in which the number of peers in a P2P network is endogenously determined by peers. There are total $N$ peers that are connected to each other, and they can form groups to share their content within a group. The maximum average individual utility increases with the number of peers in a group as shown in Proposition 3(i). Thus, in a cooperative scenario, peers will form a P2P network with all the $N$ peers if they accept a new peer as long as the inclusion of an additional peer benefits existing peers assuming that peers split total utility equally. In a non-cooperative scenario, peers do not share content at all, and thus their utilities do not depend on the number of peers. Hence, the previous results that $\Pi^{P E}=N f^{*}(\beta)$ and $\Pi^{N C}=N f^{*}(\kappa)$ are still valid with endogenous network formation. To analyze a scenario with enforced full sharing, define $\tilde{\gamma}(n)$ by $\tilde{\gamma}(n)=\kappa+(n-1) \sigma$ for $n=1,2, \ldots$. Then the average individual utility of a peer in a P2P network of size $n$ is given by

$$
g^{F S}(n)=f\left(\tilde{x}_{\tilde{\gamma}(n)}\right)-\tilde{\beta}(n) \tilde{x}_{\tilde{\gamma}(n)} .
$$

Increasing the size of a P2P network has two opposing effects on average individual utility. On one hand, increasing the number of peers benefits peers by reducing the effective marginal cost of obtaining content as represented by $\tilde{\beta}$, which decreases with $n$. On the other hand, increasing the number of peers does harm to peers by increasing the effective marginal cost of producing content as represented by $\tilde{\gamma}$, which increases with $n$. Hence, we can expect that there exists an optimal size of a P2P network that balances 
these positive and negative effects.

Since $g^{F S}(1)=f^{*}(\kappa)>0$ and $g^{F S}(n)=0$ for all $n \geq\left(f^{\prime}(0)-\kappa\right) / \sigma+1$, there must exist a maximizer of $g^{F S}(n)$ among $n=1, \ldots,\left\lfloor\left(f^{\prime}(0)-\kappa\right) / \sigma+1\right\rfloor$, denoted by $N^{*}$, where $\lfloor\alpha\rfloor$ is the largest integer smaller than or equal to $\alpha$. We assume that $N^{*}$ is unique, which will hold for a generic specification of the utility function. When $N$ peers form P2P networks endogenously to maximize their individual utilities, they will form $\left\lfloor N / N^{*}\right\rfloor$ networks of size $N^{*}$ and one network of residual peers. Hence, total utility that a coalition $\mathcal{S}$ can create is given by

$$
v^{F S}(\mathcal{S})=\left\lfloor\frac{|\mathcal{S}|}{N^{*}}\right\rfloor N^{*} g^{F S}\left(N^{*}\right)+\left(|\mathcal{S}|-\left\lfloor\frac{|\mathcal{S}|}{N^{*}}\right\rfloor\right) g^{F S}\left(|\mathcal{S}|-\left\lfloor\frac{|\mathcal{S}|}{N^{*}}\right\rfloor\right) .
$$

In order to examine the stability property of endogenous network formation, we characterize the core of the coalitional game $v^{F S}$.

Proposition 11. Suppose that $N^{*}<N$. If $N$ is a multiple of $N^{*}$, then the core of the coalitional game $v^{F S}$ consists of a unique element $v_{i}=g^{F S}\left(N^{*}\right)$ for all $i \in \mathcal{N}$. Otherwise, the core is empty.

Note that we necessarily have $N^{*}<N$ when $N \geq\left(f^{\prime}(0)-\kappa\right) / \sigma+1$. When $N$ is not a multiple of $N^{*}$, there is a residual network, whose size is smaller than $N^{*}$. A peer in the residual network can bid a utility smaller than $g^{F S}\left(N^{*}\right)$ to form a network of size $N^{*}$ including itself, yielding instability for the networks of size $N^{*}$. Suppose that $N$ is a multiple of $N^{*}$ so that the core is nonempty. The utility profile in the core is achieved by peers forming $N / N^{*}$ networks, producing $x_{i}=\hat{x}_{\tilde{\gamma}\left(N^{*}\right)} / N^{*}$ for all $i$, and sharing all produced content within a network. Social welfare at the allocation with the core property is $\Pi^{F S}=N g^{F S}\left(N^{*}\right)$. Since $g^{F S}\left(N^{*}\right) \geq g^{F S}(1)=f^{*}(\kappa)$, we have $P o N S \leq 1$ when peers can form P2P networks of the optimal size. That is, with endogenous network formation, enforced full sharing can only improve the welfare of peers because peers are given the option of operating in an autarkic manner.

\section{NUMERICAL ILLUSTRATION}

In this section, we provide illustrative results using a particular utility specification and varying the number of peers. For the utility function of peers, we use $f(x)=\log (1+x), \kappa=0.3, \delta=0.0025$, and $\sigma=0.0118$ We consider the (exogenous) number of peers in the P2P network, $N$, from 1 to 100 . Fig. 3(a) shows average individual utility in the three scenarios: $f^{*}(\beta)$ in the cooperative case, $f^{*}(\kappa)$ in the non-cooperative case, and $f\left(\tilde{x}_{\gamma}\right)-\beta \tilde{x}_{\gamma}$ in the partially cooperative case (i.e., enforced full sharing).

\footnotetext{
${ }^{18}$ The authors of [21] use the same benefit function for their illustrative examples.
} 
It can be seen that $f^{*}(\beta)$ is increasing in $N$, verifying Proposition 3(i), that $f^{*}(\kappa)$ is independent of $N$, and that $f\left(\tilde{x}_{\gamma}\right)-\beta \tilde{x}_{\gamma}$ reaches a peak at $N=5$ and is zero for all $N \geq 71$. Fig. 3[(b) plots total utility in the three scenarios: $\Pi^{P E}$ in the cooperative case, $\Pi^{N C}$ in the non-cooperative case, and $\Pi^{F S}$ in the partially cooperative case.

Fig. $3[\mathrm{c})$ compares the MP of the $n$th peer, $M P(n)$, with the maximum average individual utility that $n$ peers can achieve, $g(n)$, verifying Proposition 3(ii). Fig. 3[(d) plots the three inefficiency measures defined in Section VII. Since $f^{*}(\kappa)$ is independent of $N$, we can see that the PoA and the PoNS change with $N$ in the opposite way that $f^{*}(\beta)$ and $f\left(\tilde{x}_{\gamma}\right)-\beta \tilde{x}_{\gamma}$ change, respectively. Since $f^{*}(\beta)$ converges to $f^{*}(\delta+\sigma)=3.3945$, the PoA converges to $f^{*}(\kappa) / f^{*}(\delta+\sigma)=0.1485$ as $N$ goes to infinity. Fig. 3(e) shows the utilization of the P2P network in the three scenarios: $(N-1) \hat{x}_{\beta}$ in the cooperative case, 0 in the non-cooperative case, and $(N-1) \hat{x}_{\gamma}$ in the partially cooperative case. We can see no utilization in the non-cooperative case and underutilization (and no utilization for $N \geq 71$ ) in the partially cooperative case compared to the cooperative case, which exhibits a high utilization of the P2P network. Finally, Fig. 3[f) plots the optimal linear price $p^{*}$ as a function of $N$. As can be seen its expression in Proposition 6, $p^{*}$ decreases with $N$ and converges to $\sigma=0.01$ as $N$ goes to infinity.

\section{CONClusion And Future Directions}

In this paper, we have provided a unified framework to investigate incentive issues in content production and sharing using various game theoretic approaches. We have characterized the non-cooperative and cooperative outcomes of the CPS game and have shown that incentive schemes such as payment schemes and differential service schemes can yield a cooperative outcome among non-cooperative peers. Throughout the paper, we have discussed enforcement and information requirements to implement the solutions of different approaches with a protocol. Our analysis allows protocol designers to compare the performance and the overheads 19 of different approaches and eventually helps them select the best approach given a network environment they face.

We have maintained the homogeneity assumption in order to keep our model tractable so that we can better illustrate different approaches by providing analytic results. However, all the concepts in this paper can be straightforwardly applied to the case of peers with heterogeneous utility functions. The convexity of the coalitional game in the cooperative approach will still hold, but computing cooperative solutions will

\footnotetext{
${ }^{19}$ In this paper, we have mainly focused on communication and informational overheads, and have not addressed the issues of the complexity of computing different game theoretic solutions. Some complexity issues can be found in [39].
} 


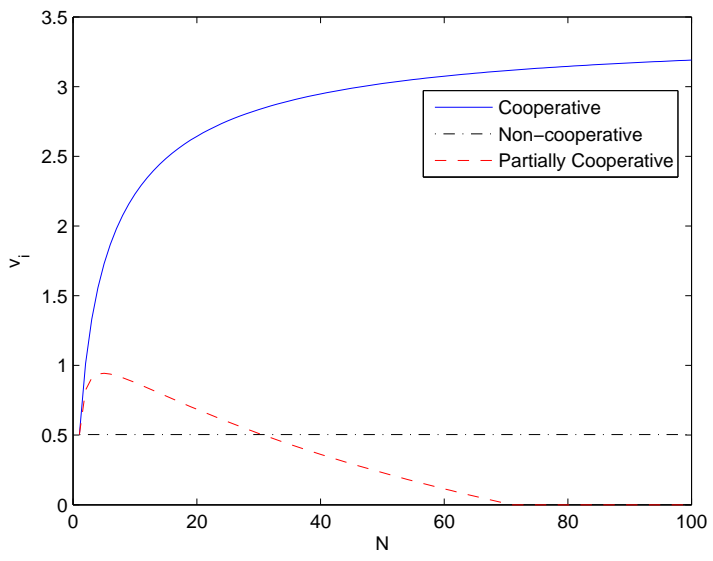

(a)

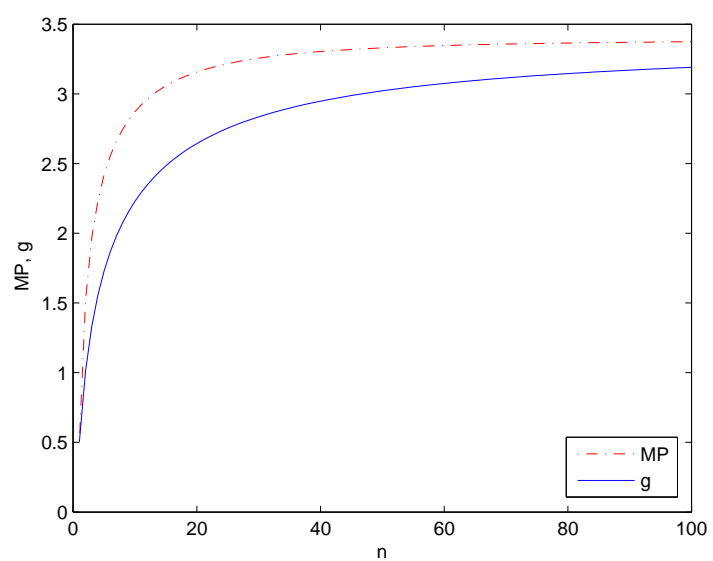

(c)



(e)

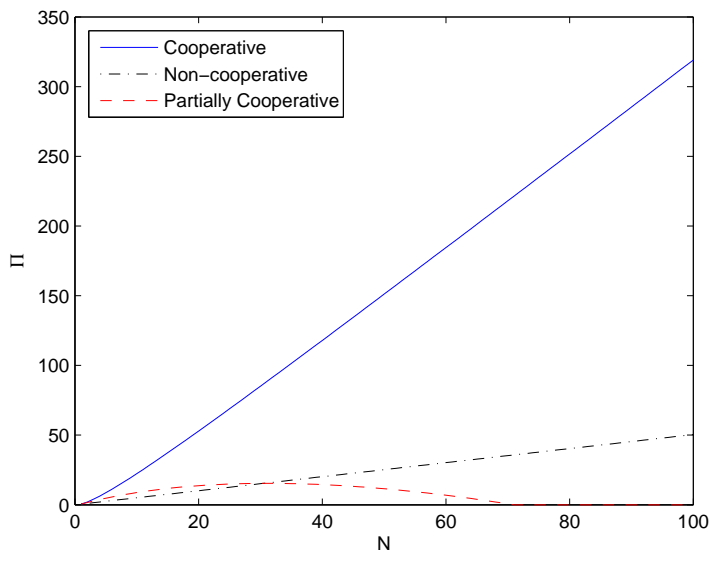

(b)



(d)



(f)

Fig. 3: Numerical results with $f(x)=\log (1+x), \kappa=0.3, \delta=0.0025, \sigma=0.01$, and varying $N$ from 1 to 100: (a) average individual utility, (b) total utility, (c) marginal product and the maximum average individual utility, (d) inefficiency measures, (e) utilization of the P2P network, and (f) optimal linear prices. 
become more complicated with heterogeneous peers. Non-cooperative solutions will remain the same with a minor change that individually optimal production levels will differ across peers. Also, heterogeneous peers in a distributed system offer a natural scenario to which a mechanism design approach can be applied. Lastly, the protocol designer may need to discriminate heterogeneous peers in order to achieve a cooperative outcome using a linear pricing scheme. Investigating how the results in this paper extend to and change in $\mathrm{P} 2 \mathrm{P}$ networks with heterogeneous peers will provide interesting and challenging future research directions.

\section{APPENDIX A}

\section{Benefit Function Proportional to the Amount of Distinct Files}

For simplicity, suppose that peers choose the number of files from the set of nonnegative integers. There are total $M$ files that can be potentially produced by a peer, where $M$ is a large positive integer. When peer $i$ produces $x_{i}$ files, it draws $x_{i}$ files with replacement from the $M$ files. (Now $\kappa$ can be considered as a constant cost of a draw.) Each file is drawn with equal probability of $1 / M$. Let $c_{i}$ be the number of files that peer $i$ consumes, i.e., $c_{i}=x_{i}+d_{i}$. Since peers cannot identify the content of files produced by others before download, $c_{i}$ files that peer $i$ consumes can be considered as $c_{i}$ independent draws from the $M$ files. The probability that a given file is not one of the $c_{i}$ files is $\left(1-\frac{1}{M}\right)^{c_{i}}$. Hence, the expected number of distinct files in the $c_{i}$ files is

$$
M\left[1-\left(1-\frac{1}{M}\right)^{c_{i}}\right]
$$

Since $\left(1-\frac{1}{M}\right)^{c_{i}} \geq 1-\frac{c_{i}}{M}$, we have

$$
M\left[1-\left(1-\frac{1}{M}\right)^{c_{i}}\right] \leq c_{i}
$$

and $c_{i}-M\left[1-\left(1-\frac{1}{M}\right)^{c_{i}}\right]$ is the expected number of redundant files in the $c_{i}$ files. If the benefit of consumption is proportional to the number of distinct files, the expected benefit function is given by

$$
f\left(c_{i}\right)=a M\left[1-\left(1-\frac{1}{M}\right)^{c_{i}}\right]
$$

for some constant $a>0$. Note that $f$ satisfies all the assumptions for a benefit function given in Section II when $a>\kappa$ if we take $c_{i}$ as a nonnegative real number rather than a nonnegative integer. In particular, $f(0)=0$ and $f$ is concave. 


\section{APPENDIX B}

\section{PROOFS OF PROPOSITIONS}

Proof of Proposition 3. (i) Note that $g(n)=f^{*}(\tilde{\beta}(n))=f^{*} \circ \tilde{\beta}(n)$. Also, $f^{*}(\alpha)=f\left(\hat{x}_{\alpha}\right)-\alpha \hat{x}_{\alpha}$, where $f^{\prime}\left(\hat{x}_{\alpha}\right)=\alpha$, for $\alpha \in\left(0, f^{\prime}(0)\right]$. That is, $\hat{x}_{\alpha}$ is the unique maximizer of $f(x)-\alpha x$ on $\mathbb{R}_{+}$. Choose $\alpha_{1}, \alpha_{2} \in\left(0, f^{\prime}(0)\right]$ such that $\alpha_{1}<\alpha_{2}$. Then $f^{*}\left(\alpha_{2}\right)=f\left(\hat{x}_{\alpha_{2}}\right)-\alpha_{2} \hat{x}_{\alpha_{2}}<f\left(\hat{x}_{\alpha_{2}}\right)-\alpha_{1} \hat{x}_{\alpha_{2}}<$ $f\left(\hat{x}_{\alpha_{1}}\right)-\alpha_{1} \hat{x}_{\alpha_{1}}=f^{*}\left(\alpha_{1}\right)$. Hence, $f^{*}$ is decreasing on $\left(0, f^{\prime}(0)\right]$. Since

$$
\tilde{\beta}(n)=\frac{1}{n}(\kappa-\delta-\sigma)+\delta+\sigma,
$$

$\tilde{\beta}$ is decreasing in $n$ and its range lies in $(\delta+\sigma, \kappa] \subset\left(0, f^{\prime}(0)\right]$. Since $g$ is a composite function of two decreasing functions, it is increasing.

Since $f$ is closed and strictly concave on $\mathbb{R}_{+}, f^{*}$ is differentiable on $\mathbb{R}_{++}[26]$. Then $f^{*}$ is continuous on $\mathbb{R}_{++}$, and thus $\lim _{n \rightarrow \infty} g(n)=\lim _{n \rightarrow \infty} f^{*}(\tilde{\beta}(n))=f^{*}\left(\lim _{n \rightarrow \infty} \tilde{\beta}(n)\right)=f^{*}(\delta+\sigma)$.

(ii) To prove that $M P$ is increasing in $n$, it suffices to show the strict convexity of $G$, taking the domain of $\tilde{\beta}$ and $G$ as $\mathbb{R}_{++}$instead of $\{1,2, \ldots\}$. Since $G(n)=n f^{*}(\tilde{\beta}(n))$ and $f^{*}$ and $\tilde{\beta}$ are differentiable on $\mathbb{R}_{++}$, by the chain rule $G$ is differentiable and

$$
G^{\prime}(n)=f^{*}(\tilde{\beta}(n))+n\left(f^{*}\right)^{\prime}(\tilde{\beta}(n)) \tilde{\beta}^{\prime}(n) .
$$

Note that $\left(f^{*}\right)^{\prime}=-\left(f^{\prime}\right)^{-1}$ on $\left(0, f^{\prime}(0)\right), f^{\prime}$ is continuously differentiable on $\mathbb{R}_{++}$, and $f^{\prime \prime}(x) \neq 0$ for all $x \in \mathbb{R}_{++}$. By the inverse function theorem, $f^{*}$ is twice continuously differentiable on $\left(0, f^{\prime}(0)\right)$, and we have

$$
G^{\prime \prime}(n)=\left(f^{*}\right)^{\prime}(\tilde{\beta}(n))\left[2 \tilde{\beta}^{\prime}(n)+n \tilde{\beta}^{\prime \prime}(n)\right]+n\left(f^{*}\right)^{\prime \prime}(\tilde{\beta}(n))\left(\tilde{\beta}^{\prime}(n)\right)^{2} .
$$

Since $2 \tilde{\beta}^{\prime}(n)+n \tilde{\beta}^{\prime \prime}(n)=0$ and $f^{*}$ is strictly convex on $\left(0, f^{\prime}(0)\right)$, we have $G^{\prime \prime}(n)=n\left(f^{*}\right)^{\prime \prime}(\tilde{\beta}(n))\left(\tilde{\beta}^{\prime}(n)\right)^{2}>$ 0 for all $n \in \mathbb{R}_{++}$. Thus, $M P(n)$ is increasing in $n$.

Note that $\operatorname{MP}(n)-g(n)=(n-1)[g(n)-g(n-1)]$ for $n \geq 2$. Since $g$ is increasing, we have $M P(n)-g(n)>0$ for all $n \geq 2$.

Since $f^{*}$ is convex and differentiable, we have

$$
\begin{aligned}
f^{*}(\tilde{\beta}(n))-f^{*}(\tilde{\beta}(n-1)) & \leq-\left(f^{*}\right)^{\prime}(\tilde{\beta}(n))[\tilde{\beta}(n-1)-\tilde{\beta}(n)] \\
& =\hat{x}_{\tilde{\beta}(n)}[\tilde{\beta}(n-1)-\tilde{\beta}(n)] \\
& <\hat{x}_{(\delta+\sigma)}[\tilde{\beta}(n-1)-\tilde{\beta}(n)] .
\end{aligned}
$$


Note that

$$
(n-1)[\tilde{\beta}(n-1)-\tilde{\beta}(n)]=\frac{1}{n}(\kappa-\delta-\sigma) .
$$

Hence,

$$
0<(n-1)[g(n)-g(n-1)]<\hat{x}_{(\delta+\sigma)} \frac{1}{n}(\kappa-\delta-\sigma)
$$

and taking limits as $n \rightarrow \infty$ yields the desired result.

Proof of Proposition 4. (i) Proposition 3(ii) implies that the coalitional game $v$ is convex. Hence, the first sentence follows from theorems 3 and 4 of [30]. The first condition for the core property, $\sum_{i \in \mathcal{N}} v_{i}(\mathbf{x}, \mathbf{y}, \mathbf{Z})=v(\mathcal{N})$, is equivalent to $\mathrm{PE}$, which requires $\sum_{i=1}^{N} x_{i}=\hat{x}_{\beta}, x_{i}=y_{i}=z_{j i}$ for all $j \neq i$, for all $i \in \mathcal{N}$ as shown in Proposition 2. Choose an arbitrary coalition $\mathcal{S}$. For a PE allocation $(\mathbf{x}, \mathbf{y}, \mathbf{Z})$, we have

$$
\sum_{i \in \mathcal{S}} v_{i}(\mathbf{x}, \mathbf{y}, \mathbf{Z})=f\left(\hat{x}_{\beta}\right)-\delta \hat{x}_{\beta}-[\kappa+(N-1) \sigma-\delta] \sum_{i \in \mathcal{S}} x_{i}
$$

Hence, using (2), we can show that (3) is equivalent to the second condition for the core property, $\sum_{i \in \mathcal{S}} v_{i}(\mathbf{x}, \mathbf{y}, \mathbf{Z}) \geq v(\mathcal{S})$.

(ii) Let $\left(v_{1}, \ldots, v_{N}\right)$ be the Shapley value of the coalitional game $v$. By the efficiency property of the Shapley value, we have $\sum_{i=1}^{N} v_{i}=v(\mathcal{N})$. Also, by the symmetry axiom, we have $v_{i}=v_{j}$ for all $i, j \in \mathcal{N}$. Combining these two yields $v_{i}=v(\mathcal{N}) / N=f^{*}(\beta)$ for all $i \in \mathcal{N}$. Using (1), we can see that $x_{i}=\hat{x}_{\beta} / N$ is necessary to obtain the Shapley value.

Proof of Proposition 5. Suppose that $d_{i}<\sum_{j \neq i} y_{j}^{e}$ for some $i \in \mathcal{N}$ at SE. Then it must be the case that $x_{i}+\sum_{j \neq i} y_{j}^{e}>\hat{x}_{\delta}$. Since $\sum_{j \neq i} y_{j}^{e} \leq \hat{x}_{\delta}$, we have $x_{i}>0$. Then we obtain a contradiction to SE because peer $i$ can improve its utility by reducing $x_{i}$ and increasing $d_{i}$ by the same amount. Thus, $z_{i j}=y_{j}^{e}$ for all $j \neq i$, for all $i \in \mathcal{N}$.

The requirement for peer $i$ that $y_{i}=y_{i}^{e}$ in stage two restricts its stage-one choice with $x_{i} \geq y_{i}^{e}$. Suppose that $x_{i}>y_{i}^{e}$ for some $i \in \mathcal{N}$ at SE. Since $d_{i}=\sum_{j \neq i} y_{j}^{e}$ at SE, the first-order effect of increasing $x_{i}$ on $v_{i}$ is given by $\partial v_{i} / \partial x_{i}=f^{\prime}\left(x_{i}+\sum_{j \neq i} y_{j}^{e}\right)-\kappa$. Since $x_{i}>y_{i}^{e}$ implies $x_{i}+\sum_{j \neq i} y_{j}^{e}>\hat{x}_{\kappa}$, we have $\partial v_{i} / \partial x_{i}<0$ for $x_{i}>y_{i}^{e}$, and thus peer $i$ becomes worse off by choosing $x_{i}>y_{i}^{e}$, contradicting SE.

Proof of Proposition 7. Let $\left(\mathbf{x}^{o}, \mathbf{y}^{o}, \mathbf{Z}^{o}\right)$ be a participation-efficient allocation. Consider the following repeated game strategy for peer $i$ : start with a cooperative strategy in the CPS game $x_{i}=x_{i}^{o}, y_{i}\left(x_{i}\right)=x_{i}$, and $\mathbf{z}_{i}\left(x_{i}, \mathbf{y}\right)=\mathbf{z}_{i}^{*}\left(x_{i}, \mathbf{y}\right)$, where $\mathbf{z}_{i}^{*}\left(x_{i}, \mathbf{y}\right)$ is the optimal download profile of peer $i$ given $\left(x_{i}, \mathbf{y}\right)$, play 
the cooperative strategy if $\mathbf{y}=\mathbf{y}^{o}$ in all the previous CPS games, and play the SE strategy of the oneshot CPS game, i.e., $x_{i}=\hat{x}_{\kappa}, y_{i}\left(x_{i}\right)=0$, and $\mathbf{z}_{i}\left(x_{i}, \mathbf{y}\right)=\mathbf{z}_{i}^{*}\left(x_{i}, \mathbf{y}\right)$, if $\mathbf{y} \neq \mathbf{y}^{o}$ in at least one of the previous CPS games. Proposition 5 implies that peer $i$ cannot gain in the current CPS game by deviating to $x_{i}>x_{i}^{o}$ or $z_{i j}<y_{j}^{o}$ for some $j \neq i$. Hence, a profitable deviation involves either $x_{i}<x_{i}^{o}$ in stage one or $y_{i}<y_{i}^{o}$ in stage two (or both). Either case results in a reduction in the sharing level from $y_{i}^{o}$. Since sharing levels are publicly observed, any profitable deviation is detectable and punishment will be triggered. Since the gain from deviation in the current CPS game is bounded above, it will be erased by the punishment in the long run. In other words, peer $i$ receives $v_{i}^{o}=v_{i}\left(\mathbf{x}^{o}, \mathbf{y}^{o}, \mathbf{Z}^{o}\right)$ on average if it follows the described repeated game strategy and $f^{*}(\kappa)$ if it deviates in a way that the deviation increases the current utility. Since $\left(\mathbf{x}^{o}, \mathbf{y}^{o}, \mathbf{Z}^{o}\right)$ is participation-efficient, we have $v_{i}^{o} \geq f^{*}(\kappa)$ for all $i \in \mathcal{N}$. Hence, the described repeated game strategy, which realizes the allocation $\left(\mathbf{x}^{o}, \mathbf{y}^{o}, \mathbf{Z}^{o}\right)$ in every CPS game, is a non-cooperative equilibrium of the repeated CPS game.

Proof of Proposition 11. Assume that the core is nonempty and choose a utility profile $\mathbf{v}=\left(v_{1}, \ldots, v_{N}\right)$ in the core. Suppose that there exists a peer $i$ with $v_{i}<g^{F S}\left(N^{*}\right)$. Consider a coalition $\mathcal{S}$ of size $N^{*}$ that do not include peer $i$, which must exist since $N>N^{*}$. Then $\sum_{j \in \mathcal{S}} v_{j}=N^{*} g^{F S}\left(N^{*}\right)$, and thus $\sum_{j \in \mathcal{S} \backslash\{k\}} v_{j} \leq\left(N^{*}-1\right) g^{F S}\left(N^{*}\right)$, where peer $k$ is the one that receives the highest utility among peers in $\mathcal{S}$. Then $\sum_{j \in(\{i\} \cup \mathcal{S} \backslash\{k\})} v_{j}<N^{*} g^{F S}\left(N^{*}\right)$, and thus peer $i$ and peers in $\mathcal{S} \backslash\{k\}$ can block the utility profile $\mathbf{v}$. Hence, we need to have $v_{i} \geq g^{F S}\left(N^{*}\right)$ for all $i \in \mathcal{N}$. This is possible, with equality, only if $N$ is a multiple of $N^{*}$. We can confirm that the core is nonempty since the utility profile $v_{i}=g^{F S}\left(N^{*}\right)$ for all $i \in \mathcal{N}$ satisfies the definition of the core.

\section{REFERENCES}

[1] H. Park and M. van der Schaar, "A framework for foresighted resource reciprocation in P2P networks," IEEE Trans. Multimedia, vol. 11, no. 1, pp. 101-116, Jan. 2009.

[2] J. Liu, S. G. Rao, B. Li, and H. Zhang, "Opportunities and challenges of peer-to-peer internet video broadcast," Proc. IEEE, vol. 96, no. 1, pp. 11-24, Jan. 2008.

[3] P. Garbacki, A. Iosup, D. Epema, and M. van Steen, "2Fast: collaborative downloads in P2P networks," in Proc. 6th IEEE Int. Conf. Peer-to-Peer Computing, 2006, pp. 23-30.

[4] J. Wang, C. Yeo, V. Prabhakaran, and K. Ramchandran, "On the role of helpers in peer-to-peer file download systems: design, analysis and simulation,” presented at the 6th Int. Workshop Peer-to-Peer Syst. (IPTPS’07), 2007.

[5] V. Vishnumurthy, S. Chandrakumar and E. G. Sirer, "KARMA: a secure economic framework for peer-to-peer resource sharing," in Proc. 1st Workshop Econ. Peer-to-Peer Syst., 2003.

[6] M. Sirivianos, J. H. Park, X. Yang, and S. Jarecski, "Dandelion: cooperative content distribution with robust incentives," in Proc. 2007 USENIX Annu. Tech. Conf., pp. 157-170. 
[7] C. Buragohain, D. Agrawal, and S. Suri, "A game theoretic framework for incentives in P2P systems," in Proc. 3rd Int. Conf. Peer-to-Peer Computing, 2003, pp. 48-56.

[8] S. D. Kamvar, M. T. Schlosser, and H. Garcia-Molina, "The EigenTrust algorithm for reputation management in P2P networks," in Proc. 12th Int. World Wide Web Conf., 2003, pp. 640-651.

[9] L. Xiong and L. Liu, "PeerTrust: supporting reputation-based trust for peer-to-peer electronic communities," IEEE Trans. Knowledge Data Eng., vol. 16, no. 7, pp. 843-857, Jul. 2004.

[10] D. Fudenberg and J. Tirole, Game Theory. Cambridge, MA: MIT Press, 1991.

[11] P. Golle, K. Leyton-Brown, I. Mironov, and M. Lillibridge, "Incentives for sharing in peer-to-peer networks," in Proc. 2nd Int. Workshop Electronic Commerce (WELCOM 2001), pp. 75-87.

[12] M. Feldman, K. Lai, I. Stoica, and J. Chuang, "Robust incentive techniques for peer-to-peer networks," in Proc. ACM Conf. Electronic Commerce (EC '04), 2004.

[13] A. Blanc, Y.-K. Liu, and A. Vahdat, "Designing incentives for peer-to-peer routing," in Proc. INFOCOM, 2005, pp. 374-385.

[14] M. Kandori, "Social norms and community enforcement," Review Econ. Stud., vol. 59, no. 1, pp. 63-80, Jan. 1992.

[15] T. B. Ma, S. C. M. Lee, J. C. S. Lui, and D. K. Y. Yau, "Incentive and service differentiation in P2P networks: a game theoretic approach," IEEE/ACM Trans. Netw., Vol. 14, No. 5, pp. 978-991, Oct. 2006.

[16] O. Loginova, H. Lu, and X. H. Wang, "Incentive schemes in peer-to-peer networks," B.E. J. Theoretical Econ., vol. 9, no. 1, Article 2, 2009.

[17] W. S. Lin, H. V. Zhao, and K. J. R. Liu, "Incentive cooperation strategies for peer-to-peer live multimedia streaming social networks," IEEE Trans. Multimedia, vol. 11, no. 3, pp. 396-412, Apr. 2009.

[18] M. K. H. Yeung and Y.-K. Kwok, "Game theoretic peer selection for resilient peer-to-peer media streaming systems," in Proc. 28th Int. Conf. Distributed Computing Syst. (ICDCS '08), 2008, pp. 817-824.

[19] H. Park and M. van der Schaar, "Coalition based Resource Negotiation for Multimedia Applications in Informationally Decentralized Networks," IEEE Trans. Multimedia, vol. 11, no. 4, pp. 765-779, Jun. 2009.

[20] J. Park and M. van der Schaar, "Pricing and Incentives in Peer-to-Peer Networks," UCLA Tech. Rep., 2009. Available at http://medianetlab.ee.ucla.edu/papers/TRP2P.pdf

[21] A. Galeotti and S. Goyal, "The law of the few," Amer. Econ. Review, to be published.

[22] M. van der Schaar, D. S. Turaga, and R. Sood, "Stochastic Optimization for Content Sharing in P2P Systems," IEEE Trans. Multimedia, vol. 10, no. 1, pp. 132-144, Jan. 2008.

[23] D. Fudenberg and D. K. Levine, “Self-confirming equilibrium ”" Econometrica, vol. 61, no. 3, pp. 523-545, May 1993.

[24] S. Saroiu, P. K. Gummadi, and S. D. Gribble, "A measurement study of peer-to-peer file sharing systems," in Proc. Multimedia Computing and Networking, 2002.

[25] E. Adar and B. A. Huberman, "Free riding on Gnutella," First Monday, vol. 5, no. 10, 2000.

[26] S. Boyd and L. Vandenberghe, Convex Optimization. Cambridge, U.K.: Cambridge Univ. Press, 2004.

[27] R. Myerson, Game Theory: Analysis of Conflict. Cambridge, MA: Harvard Univ. Press, 1991.

[28] R. Johari and S. Kumar, "Congestible services and network effects," submitted for publication.

[29] J. M. Ostroy, "A reformulation of the marginal productivity theory of distribution,” Econometrica, vol. 52, no. 3, pp. 599-630, May 1984.

[30] L. S. Shapley, “Cores of convex games,” Int. J. Game Theory, vol. 1, no. 1, pp. 11-26, Dec. 1971. 
[31] J. von Neumann and O. Morgenstern, Theory of Games and Economic Behavior. Princeton, NJ: Princeton Univ. Press, 1944.

[32] J. K. MacKie-Mason and H. R. Varian, "Pricing congestible network resources," IEEE J. Sel. Areas Commun., vol. 13, no. 7, pp. 1141-1149, Sep. 1995.

[33] F. P. Kelly, "Charging and rate control for elastic traffic," European Trans. Telecommun., vol. 8, no.1, pp. 33-37, Jan. 1997.

[34] F. P. Kelly, A. K. Maulloo, and D. K. H. Tan, "Rate control for communication networks: shadow prices, proportional fairness and stability," J. Operational Research Soc., vol. 49, no.3, pp. 237-252, Mar. 1998.

[35] R. Johari and J. N. Tsitsiklis, "Efficiency loss in a network resource allocation game," Math. Operations Research, vol. 29, no. 3, pp. 407-435, Aug. 2004.

[36] B. Cohen, "Incentives build robustness in BitTorrent," in Proc. P2P Econ. Workshop, Berkeley, CA, 2003.

[37] M. J. Osborne and A. Rubinstein, A Course in Game Theory. Cambridge, MA: The MIT Press, 1994.

[38] J. Park and M. van der Schaar, "Stackelberg contention games in multi-user networks," EURASIP J. Advances Signal Process., vol. 2009, Article ID 305978, 15 pages, 2009.

[39] N. Nisan, T. Roughgarden, É. Tardos, and V. V. Vazirani, Eds., Algorithmic Game Theory. Cambridge, U.K.: Cambridge Univ. Press, 2007. 